Chem. Phys. Lett.

Frontiers Article

$1 / 26 / 15$

\title{
Determination of the collisional energy transfer distribution responsible for the collision-induced dissociation of $\mathrm{NO}_{2}$ with $\mathrm{Ar}$
}

\author{
Jeffrey D. Steill, Ahren W. Jasper, and David W. Chandler \\ Combustion Research Facility, Sandia National Laboratories, Livermore, CA 94550, United States
}

\begin{abstract}
Collisional energy transfer is an essential aspect of chemical reactivity and maintenance of thermal equilibrium. Here we report the shape (energy-dependence) of the collisional energy transfer probability function for collisions of vibrationally excited $\mathrm{NO}_{2}$ entrained in a molecular beam and photoexcited to within $40 \mathrm{~cm}^{-1}$ of its dissociation threshold. The internally excited molecules undergo collisions with Ar atoms in a crossed beam apparatus. Dissociative collisions rapidly produce the $\mathrm{NO}(J)$ fragment, which is observed by velocity-mapped ion imaging and REMPI techniques. The measured collisional energy transfer function is obtained via energy conservation and is compared with the results of classical trajectory calculations. Good agreement between the theory and experiment is found for collisions that transfer small amounts of energy, but the theory predicts a higher likelihood of energetic collisions than is observed experimentally. We explore possible explanations for this discrepancy in the dynamics of the collision excitation process.
\end{abstract}

\section{Introduction}

Energy transfer to and from rotationally and vibrationally excited states underlies the processes of collisional stabilization, maintenance of thermal equilibrium, and pyrolysis important to chemically reactive systems such as flames. On a single chemical reaction level, the rates of many elementary reactions are pressure dependent, including unimolecular reactions that are rate-limited by energy transfer at low pressures and reactions that show falloff from their 
high-pressure limit rate coefficients at moderate pressures. Due to the central importance of energy transfer in chemical reactivity, there is a long history of studies of collisional energy transfer of highly excited molecules. Almost 20 years ago, Flynn, Parmenter, and Wodtke reviewed the vast vibrational energy transfer literature and provided 465 references $^{1}$ including earlier reviews; ${ }^{2,3,4}$ numerous relevant papers have appeared since then.

Investigations involving both activating and deactivating collisions have been performed, but, in general, the study of collisional deactivation of highly vibrationally excited species has been more prevalent. Experimental information about collisional energy transfer can be obtained indirectly from low-pressure-limit unimolecular decomposition and isomerization kinetics, and such studies have been carried out for several small unimolecular systems in different atomic and molecular baths. ${ }^{5-8}$ More direct experimental studies of collisional energy transfer have also appeared. These have focused on characterizing the energy transfer function $P\left(E, E^{\prime}\right)$, which describes the probability that a collision changes the internal energy of the unimolecular species from some internal state $E^{\prime}$ to some other state $E$. In some studies, low-order moments of $P\left(E, E^{\prime}\right)$ have been obtained by following the time-dependent energy content either of the initially excited molecule or the (bath gas) collision partner, including the time-dependent IR emission studies of Barker $^{9,10}$ and Dai ${ }^{11,12}$ and the time-resolved UV absorption spectroscopy experiments of Troe and co-workers. ${ }^{13,14}$ The work of Lenzer and Luther, through their kinetically controlled selective ionization studies, improved this overall approach by giving information about not only the average energy transferred but the shape of the $P\left(E, E^{\prime}\right)$ distribution ${ }^{15,16}$ and reconciled historical discrepancies ${ }^{17}$ between the earlier studies. Their work demonstrated that there is an energy dependence to both the first and second moment of the energy transfer distribution (the average energy transferred and the width of the energy transfer distribution). Their work also suggested the presence of "supercollisions," low-probability events involving a very large degree of rovibrational/translational energy transfer, first predicted by Miller and Brown. ${ }^{18}$ It is accepted that most $P\left(E, E^{\prime}\right)$ curves from vibrationally hot polyatomic molecules are better described by a double exponential (or a stretched exponential) than a single exponential. The strong collisions that represent approximately $10 \%$ or less of the collisions that make up the long "supercollision" tail represent up to $25 \%$ of the energy transfer in some systems.

Supercollisions were first detected experimentally by Flynn and co-workers through high-resolution, diode laser transient absorption spectroscopy on collisionally excited 
(rotationally and translationally) $\mathrm{CO}_{2}$ as a collision partner of a very vibrationally hot perfluorobenzene molecule. ${ }^{19}$ These experiments are sensitive to the strong collisions that transfer large amounts of energy to the rotational and translational degrees of freedom of the $\mathrm{CO}_{2}$ collision partner. As the experiment is performed in a bulb gas arrangement the $\mathrm{CO}_{2}$ has an initial thermal distribution and therefore small amounts of energy transfer are not observed. These studies have been expanded by the Mullin ${ }^{20,21}$ and Sevy ${ }^{22}$ groups to many systems with either water or $\mathrm{CO}_{2}$ as a collider gas. These experiments provide information on the energy transfer of collisions transferring approximately $1500 \mathrm{~cm}^{-1}$ or larger. Great strides in understanding these energetic collisions for large benzene like molecules have been made with the development of the "GRETCHEN" model $^{23,24}$ which has been able to demonstrate a strong correlation between the energy transfer for collisions transferring at least $E>3000 \mathrm{~cm}^{-1}$ with the density of states of the molecule in accord with Fermi's Golden Rule. ${ }^{25}$ From trajectory calculations on these systems, there remains uncertainty about the exact nature of the collisions with some calculations indicating that certain modes promote energetic collisions, ${ }^{26}$ sometimes involving chattering collisions and complex formation, ${ }^{27}$ some suggesting special collisions where a $\mathrm{H}$ atom was pinched between the backbone of the molecule and the collider molecule ${ }^{28}$ were important, and others suggesting that rotational energy transfer of large torque were responsible for the high energy wing of the distribution. ${ }^{29,30}$

Cold supersonic expansions for production of molecular beams provides a way to achieve greater state selectivity in the degree of internal excitation, as was shown by the experiments of Parmenter and co-workers that investigated vibrational energy transfer of excited electronic states $^{31,32}$. By employing two crossed-molecular beams for the collision experiments the advantage of initial state selectivity is coupled with well-defined large collision energies. Due to the selective excitation to a single ro-vibrational state in the excited electronic state, these crossed-beam experiments have the advantage of being able to observe both vibrational deactivating and activating collisions. These studies showed that even in very large molecules like p-difluorobenzene with large amounts of vibrational excitation transfers involving single quantum changes in the lowest frequency mode, $v^{\prime}(30)=120 \mathrm{~cm}^{-1}$, are the dominant single channels for all levels. That is, the identity of the quantum states remains important to the energy transfer. 
The first crossed-beam studies of vibrationally excited molecules that focused upon collisions of very vibrationally hot ground state molecules were those of Fisk and co workers in the 1970's ${ }^{33,34,35}$ who studied energy transfer from hot KBr. More recently two groups have used crossed molecular beam techniques to study vibrationally hot ground state molecules. In an experimental arrangement very similar to the one we use here, Reisler and co-authors have also investigated collisional excitation of highly-excited $\mathrm{NO}_{2} \cdot{ }^{36,37,38}$ These will be described in some detail below as they are to be compared with the results we obtain here. Ni and co-workers ${ }^{39,40}$ also used collisions in crossed supersonic atomic and molecular beams to measure the single collision energy transfer between azulene and rare gas atoms. By ionizing the hot azulene that has had a collision and measuring its velocity distribution utilizing velocity mapped sliced imaging they were able to make a measurement of the shape of the energy transfer function, $P\left(E, E^{\prime}\right)$. Subsequent trajectory calculations of this distribution were disappointingly unable to match the experiment as the calculations predicted only a third of the energy transfer as the experiments measured. $^{41}$

The $\mathrm{NO}_{2}$ molecule provides an important benchmark system due to the plethora of investigations into the collisional and photodissociation dynamics of the molecule. $\mathrm{NO}_{2}$ is a molecular system with a facile means for excitation and the NO products are easily detected by resonant ionization of near UV light $(\sim 226 \mathrm{~nm})$. While it is easy to produce a molecule with a low degree of vibrational excitation one cannot generally produce a molecule vibrationally excited to within a few wavenumbers below its dissociation limit. However, $\mathrm{NO}_{2}$ can be excited to a highly vibrationally excited state directly through one photon excitation to an excited electronic state followed by internal conversion of this electronically excited state to the ground state. The details of collisional energy transfer to $\mathrm{NO}_{2} *$ have previously been studied both in crossed-beam and bath-gas experiments, such as the crossed-beam experiments Reisler and coworkers mentioned above, ${ }^{36}$ as well as a series of bath-gas experiments performed by Dai and coworkers, ${ }^{11,12,42}$ and a state-to-state study by Abel et al. ${ }^{43}$ that examined $\mathrm{NO}_{2}+\mathrm{NO}_{2}$ collisional energy transfer. Like the present experiments reported here, the experiments of Reisler and coworkers utilized collision induced dissociation followed by detection of the dissociation product, $\mathrm{NO}(J)$, to monitor the energy transfer. ${ }^{36}$ They were able to observe and quantify the shape of the high energy tail of the energy transfer function associated with energy transfer greater than $\sim 300$ $\mathrm{cm}^{-1}$. Our current study, utilizing a different detection scheme, is complementary to Reisler's 
study as it quantifies the lower energy region of the energy transfer function, i.e. energies lower than $\sim 300 \mathrm{~cm}^{-1}$, and is sensitive to the shape of the distribution as well. There is a small region of overlap between the two studies.

Collisional energy transfer in highly vibrationally excited molecules has been studied extensively using classical trajectory calculations. These calculations can yield detailed information on the collisional energy transfer processes, ${ }^{18,44-54}$ but their accuracy can be limited by the quality of the potential energy surfaces. Examples of recent studies include the collisional energy transfer calculations of Barker et al., that examined the degree of excitation and deexcitation of polyatomics in collisions with $\mathrm{Ar}^{55}$ the collisional stabilization calculations of Babikov and co-workers, ${ }^{56}$ and the allyl + Ar trajectory studies of Conte et al. ${ }^{57,58}$ The accuracy of trajectory-based collisional energy transfer calculations can be inferred by comparing predicted low-pressure-limit and pressure-dependent rate coefficients - which are sensitive to collisional energy transfer-with available experimental results. ${ }^{59-62}$ Such comparisons are indirect, however. Differences in the theoretical and measured kinetics may arise due to other aspects of the calculation. Here, we compare experimental and computed results for the detailed energy transfer function, $P\left(E, E^{\prime}\right)$, which is much more direct test of the trajectory methods than comparisons of highly averaged kinetics. As noted above, a similar comparison of $P\left(E, E^{\prime}\right)$ for azulene + Ar showed qualitative, but not quantitative, agreement between the theoretical and experimental results. ${ }^{3940-41}$ The magnitude of this discrepancy is perhaps surprising in light of the very accurate low-pressure kinetics predictions obtained using models that depend sensitively on trajectory-based collisional energy transfer. ${ }^{62}$

Here we consider the shape (i.e., the $E$-dependence) of the collisional energy transfer function $P\left(E, E^{\prime}\right)$. This function is often approximated by an energy-gap or exponential-gap law, where $P\left(E, E^{\prime}\right)=A \exp (-\Delta E / \alpha), \Delta E=E-E^{\prime}$, and $\alpha$ is related to the collision efficiency. Trajectory calculations nearly universally show a more complicated shape for this function, one that can be better modeled by a multiexponential decay function. ${ }^{18}$ With this in mind, we utilize a novel experimental strategy for measuring the shape of the energy transfer distribution, $P\left(E, E^{\prime}\right)$, of highly vibrationally excited $\mathrm{NO}_{2}$ molecules in a collision with Ar atoms. The measured shape of the energy transfer function is compared with the results of classical trajectory calculations. 


\section{Methods}

\subsection{Experimental}

The crossed-beam velocity-mapped ion imaging (VMII) apparatus utilized for the current experiment has been described previously. ${ }^{63}$ As shown in the spatial schematic in panel (a) of Fig. 1, a pulsed nozzle jet expansion of pure Ar is crossed at 90 degrees with an expansion of approximately $15 \% \mathrm{NO}_{2}$ entrained in $\mathrm{Ar}$, with $5 \% \mathrm{O}_{2}$ added to reduce the amount of $\mathrm{NO}$ in the beam, to investigate single-collision dynamics of $\mathrm{NO}_{2}$ with Ar. The $\mathrm{NO}_{2}$ molecules are photoexcited before the collisions by a pulsed dye laser with a $\lambda_{\text {pump }}$ of $398.5 \mathrm{~nm}$. The scattering products are probed with a second pulsed dye laser with a $\lambda_{\text {probe }}(\sim 226 \mathrm{~nm})$ on the $\mathrm{A}-\mathrm{X}$ transition of the $\mathrm{NO}(J)$ fragmentation products of the Ar collision with the excited $\mathrm{NO}_{2}$. The $\mathrm{NO}_{2}$ is photoexcited before the crossing region of the atomic and molecular beams, as shown in Fig 1. panel (a). Introducing a spatial separation between the photoexcitation and scattering center prevents the observation of photodissociation products of collisionally-excited ground-state $\mathrm{NO}_{2}$ molecules that would be excited by the $\lambda_{\text {pump }}$.

The preparation of our highly vibrationally excited $\mathrm{NO}_{2}$ molecules follows the scheme utilized by previous studies. ${ }^{11,36}$ Laser excitation of jet expansion-cooled $\mathrm{NO}_{2}$ to the $\mathrm{NO}_{2}(\mathrm{~A} / \mathrm{B})$ state results in internal conversion to the overlapping $\mathrm{NO}_{2}(\mathrm{X})$ ground electronic state. As shown in the schematic in panel (b) of Fig. 1 the laser excitation of the NO2 to a highly vibrational excited state $\left(\mathrm{NO}_{2}{ }^{*}\right)$ with a $\lambda_{\text {pump }}$ of $398.6 \mathrm{~nm}\left(25088 \mathrm{~cm}^{-1}\right)$ is $40 \mathrm{~cm}^{-1}$ below the dissociation threshold, $D_{\mathrm{e}}$. The dissociation threshold for $\mathrm{NO}_{2}(\mathrm{X})$ is $25128 \mathrm{~cm}^{-1} \cdot{ }^{64,65}$

At the beam velocities employed, a collision of $\mathrm{NO}_{2} *$ with $\mathrm{Ar}$ has sufficient energy to promote the $\mathrm{NO}_{2} *$ to a ro-vibrationally excited state, $\mathrm{NO}_{2}{ }^{* *}$, above the dissociation threshold. The barrierless dissociation of $\mathrm{NO}_{2}(\mathrm{X})$ produces fragments in their electronic ground state: $\mathrm{NO}\left({ }^{2} \Pi\right)$ molecules and $\mathrm{O}\left({ }^{3} \mathrm{P}\right)$ atoms. The collisionally produced $\mathrm{NO}(J)$ fragments are detected by REMPI ionization. This scheme is illustrated by the REMPI spectrum and schematic in panel (b) of Fig. 1. By tuning the wavelength $\lambda_{\text {probe }}$ around $226 \mathrm{~nm}$, specific rotational states of the NO fragment distributions are ionized. The ionization is performed in the ion lens of the VMII apparatus in order to measure the velocity distribution of the rotationally state selectively ionized NO fragments. The resultant velocity distribution of the NO fragments is the convolution of the original $\mathrm{Ar}+\mathrm{NO}_{2} *$ scattering dynamics and the subsequent $\mathrm{NO}_{2} * * \rightarrow \mathrm{NO}(J)+\mathrm{O}$ dissociation dynamics, as discussed in detail below. 
The Newton diagram schematic of panel (c) in Fig. 1 illustrates the relative components in velocity space. The nozzle jet expansion of $\mathrm{NO}_{2}$ entrained in Ar results in a velocity distribution with a mean of $560 \mathrm{~m} / \mathrm{s}$ and a FWHM of $100 \mathrm{~m} / \mathrm{s}$. The same velocity distribution characteristics are measured for the neat Ar beam by seeding a small amount of NO into the Ar beam and using the VMII apparatus to determine the velocity and velocity spread. At a collision angle of 90 degrees, these velocities result in a collision energy distribution with a mean of 560 $\mathrm{cm}^{-1}$ and a FWHM of $140 \mathrm{~cm}^{-1}$. The elastic scattering of $\mathrm{NO}_{2} *$ shown by the large gray circle demonstrates the conservation of all kinetic energy in either $\mathrm{NO}_{2} *$ or Ar velocities. Only the collisions which impart internal energy to the $\mathrm{NO}_{2} *$ molecule by removal of some of the total kinetic energy to make $\mathrm{NO}_{2}{ }^{* *}$ are capable of producing fragment $\mathrm{NO}(J)$ molecules that are imaged. A single velocity map ion image of a given $\mathrm{NO}(J)$ state is acquired by averaging over many laser pulses. Depending upon signal intensity, averages were accumulated over 5 to 10 hours utilizing $30 \mathrm{~Hz}$ lasers.

At the collision energies of our experiment no vibrational excitation of the NO fragment is possible, only rotational excitation. Thus, the collection of the energetically possible $\operatorname{NO}(J)$ states provides a full record of the collisional energy transfer distribution since the dissociated $\mathrm{NO}(J)$ and $\mathrm{O}$ fragments are momentum-matched. While the pump laser provides a very narrow distribution of $\mathrm{NO}_{2} *$ excitation energies, there is some additional spread in the degree of $\mathrm{NO}_{2} *$ excitation due to incompletely cooled $\mathrm{NO}_{2}$ in the beam. The nozzle-jet expansion of $\mathrm{NO}_{2}$ in $\mathrm{Ar}$ resulted in a $\mathrm{NO}_{2}$ rotational temperature of $\sim 25 \mathrm{~K}$. This was determined by scanning the pump laser through the dissociation threshold for various $J$-states of NO. 


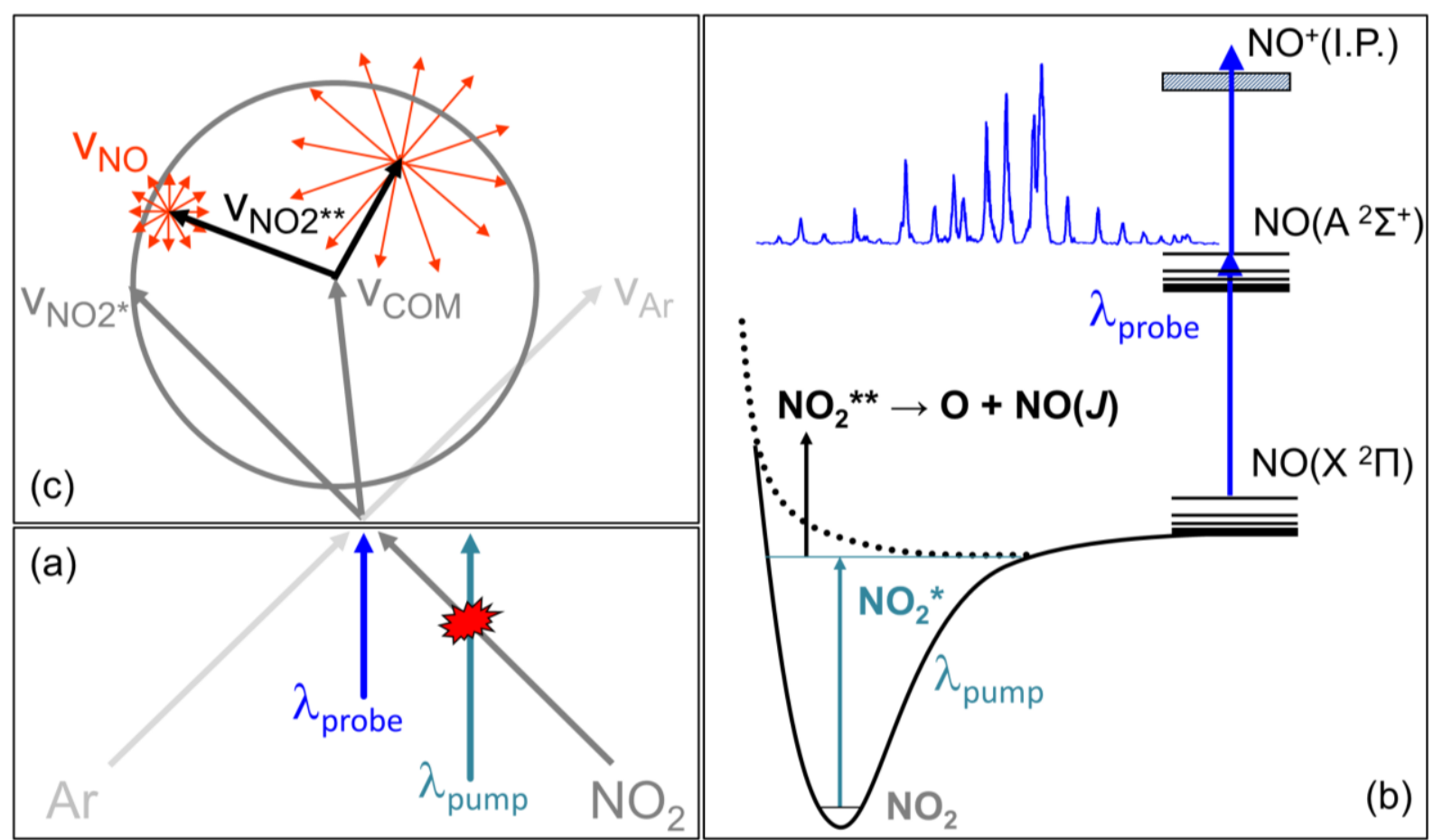

Figure 1. Schematic representation of experiment. Panel (a) shows the spatial configuration, panel (b) shows the relative energetics, and panel (c) illustrates the key velocity components of the experiment. The Newton diagram shown in panel (c) shows the velocity components of the NO fragment as emanating from the velocity vector of the inelastically scattered $\mathrm{NO}_{2} * *$ molecule; the dynamics of $\mathrm{NO}_{2} *+$ Ar scattering are centered about the center-of-mass velocity, $v_{\mathrm{COM}}$.

\subsection{Theory}

The collisional energy transfer function $P\left(E, E^{\prime}\right)$ for ground state $\mathrm{NO}_{2}$ colliding with $\mathrm{Ar}$ was calculated using a newly fitted potential energy surface (PES) and classical trajectories. The PES for $\mathrm{NO}_{2}+$ Ar may be written

$$
V=V_{\mathrm{NO} 2}+V_{\mathrm{I}}
$$

where $V_{\mathrm{NO} 2}$ is the PES for isolated $\mathrm{NO}_{2}$, and $V_{\mathrm{I}}$ is the intermolecular $\mathrm{NO}_{2}-\mathrm{Ar}$ PES. Several fitted $\mathrm{NO}_{2}$ potential energy surfaces have appeared in the literature. ${ }^{66-69}$ A new analytic representation of $V_{\mathrm{NO} 2}$ was obtained here by fitting a simple functional form to the results of dynamically weighted (dw) Davidson-corrected (+Q) multireference configuration interaction (dwMRCI+Q/CBS) calculations. ${ }^{70,71}$ The dw scheme included six electronic states and a range parameter of $2 \mathrm{eV}$. The (9e,7o) active space was used, and the complete basis set (CBS) limit was extrapolated via a two-point formula from the cc-pVTZ and cc-pVQZ basis sets. This 
method predicts an equilibrium geometry of $\mathrm{NO}_{2}$ that is in very good agreement with experiment, ${ }^{72}$ with $\mathrm{N}-\mathrm{O}$ distances differing by less than $0.001 \AA$ and an $\mathrm{O}-\mathrm{N}-\mathrm{O}$ angle differing by less than $1^{\circ}$. The energies of the optimized geometries produce a classical $\mathrm{ON}-\mathrm{O}$ dissociation energy $\left(25617 \mathrm{~cm}^{-1}\right)$ that is in reasonable agreement with the value inferred from experiments by Bezel et al. ${ }^{64}\left(25128 \mathrm{~cm}^{-1}\right)$. The use of a multireference method allows for an accurate characterization of the $\mathrm{NO}_{2}$ PES globally, including energies near the threshold for bond breaking, as demonstrated previously for $\mathrm{CO}_{2}{ }^{73}$ The calculated dw-MRCI+Q/CBS energies included geometries with $\mathrm{N}-\mathrm{O}$ distances varying from $0.7-5 \AA$ and $\mathrm{O}-\mathrm{N}-\mathrm{O}$ angles varying from $45-180^{\circ}$. Geometries with energies above $50,000 \mathrm{~cm}^{-1}$ were excluded from the data set (where the zero of energy was set to the classical equilibrium for $\mathrm{O}+\mathrm{NO}$ ), resulting in a final data set of 6684 geometries.

The data set was symmetrized with respect to O-atom exchange, doubling its size. These data were fit to the functional form

$$
V_{\mathrm{NO}_{2}}=V_{\mathrm{NO}}\left(R_{1}\right)+V_{\mathrm{NO}}\left(R_{2}\right)+c_{i, j, k} c_{i j k} x_{1}^{i} x_{2}^{j} x_{3}^{k},
$$

where $R_{1}$ and $R_{2}$ are the two N-O distances, $V_{\text {NO }}$ is a one-dimensional NO PES, $R_{3}$ is the O-O distance,

$$
x_{a}=\exp \left(R_{a}\right),
$$

$i, j, k=0-7$, and the 343 linear coefficients $c_{i j k}$ were determined via a weighted least-squares minimization. Geometries with energies below $-15000 \mathrm{~cm}^{-1}$ were weighted more heavily than the other data. The mean unsigned error over the entire data set was $750 \mathrm{~cm}^{-1}$, and the mean unsigned error for data with energies below $-15000 \mathrm{~cm}^{-1}$ was $210 \mathrm{~cm}^{-1}$. The equilibrium structure for $\mathrm{NO}_{2}$ on the fitted surface is within $0.002 \AA$ and $0.2^{\circ}$ of the experimental values, with a classical dissociation energy of $25734 \mathrm{~cm}^{-1}$. Calculated rotational constants for the fitted surface $\left(7.95,0.439,0.416 \mathrm{~cm}^{-1}\right)$ are in excellent agreement with experiment ${ }^{72}(8.00,0.433$, $\left.0.410 \mathrm{~cm}^{-1}\right)$. Agreement is also good for the fitted $\left(809,1404,1581 \mathrm{~cm}^{-1}\right)$ and experimental harmonic frequencies ${ }^{72}\left(750,1318,1618 \mathrm{~cm}^{-1}\right)$.

The "separable pairwise" approximation was used for $V_{\mathrm{I}}$, where

$$
V_{\mathrm{I}}=\sum_{a} A_{a} \exp \left(-R_{a} / B_{a}\right)-\left(C_{a}^{6} /\left(R_{a}^{6}+D_{a}^{6}\right)\right)
$$


and the index $a$ labels the three intermolecular pairs of atoms (the $\operatorname{Ar}-\mathrm{N}$ and the two $\operatorname{Ar}-\mathrm{O}$ distances). The eight unique adjustable parameters in eq 4 (the same parameters were used for both Ar-O interactions) were fit to 169 counterpoise corrected QCISD(T)/CBS energies for $\mathrm{NO}_{2}$ $+\mathrm{Ar}$, where the CBS limit was estimated using a two-point formula with the aug-cc-pVDZ and aug-cc-pVTZ basis sets, with a mean unsigned error of $10 \mathrm{~cm}^{-1}$. For fixed $\mathrm{NO}_{2}-\mathrm{Ar}$ orientations, the local minima in the interaction energies vary from -90 to $-180 \mathrm{~cm}^{-1}$ and the $\mathrm{Ar}-\mathrm{N}$ distances associated with these minima vary from 3.4-4.6 $\AA$. This anisotropy and the anisotropy of $V_{\mathrm{I}}$ at shorter, repulsive distances are well reproduced in the fitted surface. As demonstrated by numerous classical trajectory studies[46,49-52,74,75], energy transfer is most sensitive to the repulsive part of $V_{\mathrm{I}}$ and is relatively insensitive to the PES of the target species ( $\left.V_{\mathrm{NO2}}\right)$. The present PES fitting strategy has been validated using full-dimensional direct dynamics trajectories[60,76], and has been used in quantitative dynamical studies where the results are expected to depend sensitively on $V_{\mathrm{I}}$, including predictions of the unimolecular kinetics of $\mathrm{CH}_{4}$ and $\mathrm{C}_{2} \mathrm{H}_{3}+\mathrm{He}$ [62] and predictions of dilute gas diffusion coefficients for several $\mathrm{n}$-alkanes + $\mathrm{N}_{2}$. [77]

$\mathrm{NO}_{2}+\mathrm{Ar}$ collisions were simulated using classical trajectories where the initial vibrational (and total) energy of $\mathrm{NO}_{2}, E^{\prime}$, was set to $97 \%$ of the classical dissociation energy with no initial rotational energy, $J^{\prime}=0$. The initial coordinates and momenta were obtained for the $E^{\prime}, J^{\prime}$ microcanonical ensemble by sampling uniformly from separate long-time trajectories for isolated $\mathrm{NO}_{2}$. The relative collision energy for each trajectory was selected from a Gaussian distribution with a center at $560 \mathrm{~cm}^{-1}$ and a width parameter of $60 \mathrm{~cm}^{-1}$. The impact parameter was sampled out to very long range $\left(b_{\max }=10 \AA\right)$, and the initial and final $\mathrm{NO}_{2}-\mathrm{Ar}$ separations were $14 \AA$. At these large final separations, the total energy of $\mathrm{NO}_{2}$ after the collision, $E$, can be calculated unambiguously, and the energy transferred in the collision is defined $\Delta E=E-E^{\prime}$. We also calculated the final rotational state of $\mathrm{NO}_{2}, J$. The vibrational energy transferred in the collision can be defined $\Delta E_{\mathrm{vib}}=\Delta E-B J^{2}$, where $B=0.42 \mathrm{~cm}^{-1}$ and $B J^{2}$ is the final classical rotational energy of $\mathrm{NO}_{2}$. The ensemble consisted of 120000 trajectories.

The choice of initial vibrational energy in the trajectory calculations is further from the threshold for dissociation than in the accompanying experiments, but it avoids the complication of characterizing the final states of dissociated $\mathrm{NO}_{2}$. We tested the sensitivity of our calculated results to the choice of $E^{\prime}$ (where $E^{\prime}$ was set instead to $91 \%$ or $94 \%$ of the dissociation energy) 
and for ensembles where $J^{\prime}$ was selected from a classical thermal distribution with a rotational temperature of $15 \mathrm{~K}$. The results of these tests agreed quantitatively with the results presented here.

\section{Results and Discussion}

In order to determine the shape of the collisional energy transfer probability distribution, $P\left(E, E^{\prime}\right)$, we measure the velocity distribution of the NO fragments that result form collisions that have sufficient energy to produce different rotational states of the NO. From the velocity of these fragments and knowledge about how the collisionally excited $\mathrm{NO}_{2} * *$ molecule dissociates when excited to a particular energy we are able to extract from the data the shape of the original collisional energy transfer probability distribution between the $\mathrm{NO}_{2} *$ and the Ar.

The following three-step mechanism describes the collisional induced dissociation processes. The related energies are depicted in Figure 2.

$$
\begin{gathered}
\mathrm{NO}_{2}+h v(398.6 \mathrm{~nm}) \rightarrow \mathrm{NO}_{2} * \\
\mathrm{NO}_{2}^{*}+\mathrm{Ar} \rightarrow \mathrm{NO}_{2}^{* *}+\mathrm{Ar} \\
\mathrm{NO}_{2}^{* *} \rightarrow \mathrm{NO}(J)+\mathrm{O}
\end{gathered}
$$

The first step is a photoexciation of ground state $\mathrm{NO}_{2}$ to internally vibrationally excited state, $\mathrm{NO}_{2}{ }^{*}$, with an energy denoted $E_{\text {int }}$. The second step represents an inelastic scattering event that increases the internal energy of the $\mathrm{NO}_{2} *$ to a value by an amount denoted $E_{\mathrm{txfr}}$ above the dissociation threshold; the conversion of kinetic to internal energy produces an $\mathrm{NO}_{2} * *+\mathrm{Ar}$ system with a reduced total kinetic energy denoted as $E_{\text {trans. }}$. The final process represents the unimolecular dissociation of the $\mathrm{NO}_{2}{ }^{* *}$ molecule to specific rotational states of $\mathrm{NO}$ with associated kinetic energy releases, denoted $E_{\text {recoil }}$, that are determined by the rotational energy, $\mathrm{E}_{\mathrm{rot}}$, and the inelasticity of the collision (step 2). The relationship of these energies is described in detail below to explain how the NO velocity distribution provides a direct metric of the collisional energy transfer probability distribution. This three step mechanism contains within it the assumption that the Ar atom does not participate in the $\mathrm{NO}^{* *}$ dissociation event. We will examine this assumption below.

The principles of conservation of energy and momentum underpin the analysis of the NO images. The distribution of NO velocities is related to the system energetics as shown in Figure 2. In this experiment what is measured is the velocity distribution of a particular $\operatorname{NO}(J)$ rotational 
state, and due to the knowledge of all the other terms, this velocity distribution is directly related to the $\mathrm{NO}_{2}+\mathrm{Ar}$ collisional energy transfer distribution. As illustrated in Figure 2, the NO fragment velocities are a vector sum of two components: the $\mathrm{NO}_{2}{ }^{* *}$ "translation" velocities (i.e., the velocity of the $\mathrm{NO}_{2} * *$ molecule after the collision that together with the Ar collision partner constitutes the kinetic energy that was not converted to internal energy) and the "recoil" velocity, which is from the portion of the excess energy in $\mathrm{NO}_{2}{ }^{* *}$ above the dissociation threshold that results in $\mathrm{NO}$ and $\mathrm{O}$ kinetic energy release. By analysis of the energy relationships we see that the magnitudes of the recoil and "translational" velocities are interrelated at a given $\mathrm{NO}(J)$ rotational state.

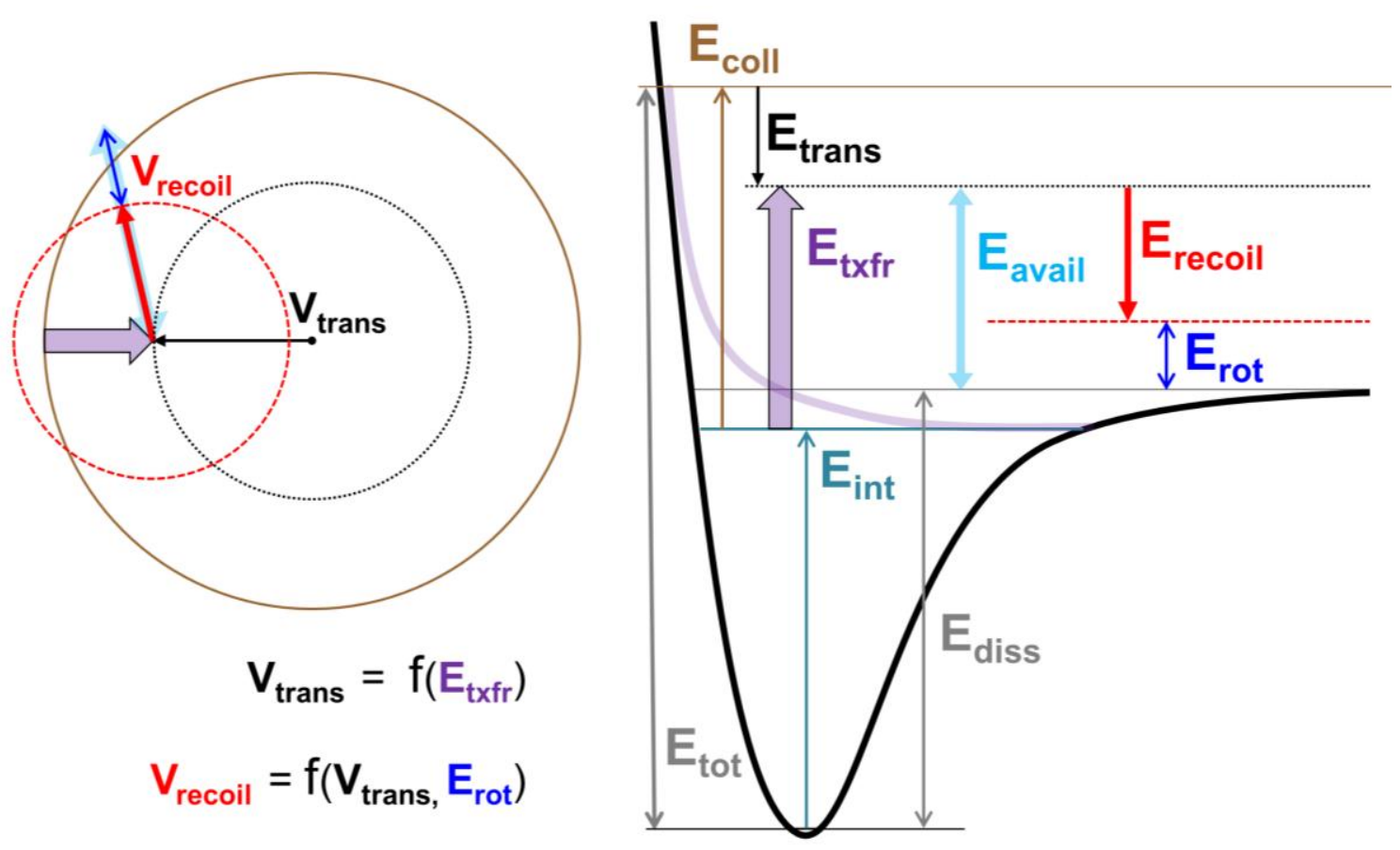

Figure 2. Schematic representation of energy and momentum conservation approach of experiment. See text for definition of terms, and online edition for references to color. The distribution of energies transferred from kinetic to internal energy of $\mathrm{NO}_{2}, E_{\mathrm{txfr}}$, is determined by the measurement of the NO velocity distribution and rotational state. The NO velocity is a vector sum of two components, the recoil velocity from the $\mathrm{NO}_{2}{ }^{* *}$ dissociation event and the velocity of the $\mathrm{NO}_{2}{ }^{* *}$ that comes from the unused "translational" kinetic energy of the $\mathrm{NO}_{2}+\mathrm{Ar}$ system. The inelastically scattered $\mathrm{NO}_{2}{ }^{* *}$ molecule undergoing dissociation is shown as forward-scattered, where in the experiment the red (dashed-line) recoil "rings" of $v_{\text {recoil }}$ can emanate from all possible positions on the correlated black (dotted-line) "origin" set by $v_{\text {trans. }}$. Note that for a given amount of collisional excitation in $\mathrm{NO}_{2}{ }^{* *}, v_{\text {recoil }}$ is reduced by the NO rotational energy. 
A series of energy relationships constrain the energy available for $\mathrm{NO}+\mathrm{O}$ kinetic energy release $\left(E_{\text {recoil }}\right)$. As shown in the schematic of Fig. 2, the total energy of the system $\left(E_{\mathrm{tot}}\right)$ is the sum of the internal energy of the vibrationally excited $\mathrm{NO}_{2} *$ molecule $\left(E_{\text {int }}\right)$ and the collision energy $\left(E_{\text {coll }}\right)$.

$$
E_{\mathrm{tot}}=E_{\mathrm{int}}+E_{\mathrm{coll}}
$$

The internal energy ( $E_{\text {int }}$ ) of the $\mathrm{NO}_{2} *$ molecule is set by the $\lambda_{\text {pump }}$ and is accurately and precisely measured, the collision energy $E_{\text {coll }}$ is set by the beam velocities and is accurately determined with a known distribution. The unknown, $E_{\mathrm{txf}}$, is the amount of energy available in the collision that is removed from the total kinetic energy of the collision partners and transferred to the internal degrees of freedom of the $\mathrm{NO}_{2} *$ to form $\mathrm{NO}_{2}{ }^{* *}$. This $E_{\mathrm{txfr}}$ distribution is shown schematically by the exponential decay function at an energy of $E_{\text {int. }}$. For illustration, a single $\mathrm{E}_{\mathrm{txfr}}$ value is shown schematically and the remaining energies (except $E_{\text {diss, }}$, the known $\mathrm{NO}_{2}$ dissociation energy) are referenced to this quantity. A given value of $E_{\mathrm{txfr}}$ leaves a given amount of "translational" kinetic energy ( $\left.E_{\text {trans }}\right)$ in the system corresponding to the remaining collision energy that was not used to increase the internal energy of $\mathrm{NO}_{2} *$ to make $\mathrm{NO}_{2} * *$.

$$
E_{\mathrm{txfr}}=E_{\mathrm{coll}}-E_{\text {trans }}
$$

Of the $E_{\mathrm{txfr}}$ energy that is input into the $\mathrm{NO}_{2}{ }^{* *}$ system, a certain amount is required to push the $\mathrm{NO}_{2} *$ molecule above the dissociation threshold, and only the excess energy $E_{\text {avail }}$ is available for the $\mathrm{NO}$ and $\mathrm{O}$ fragments. The available energy $E_{\text {avail }}$ is a function of the difference between the internal energy of the $\mathrm{NO}_{2}{ }^{* *}$ and the $\mathrm{NO}_{2}$ dissociation energy, i.e.,

$$
E_{\text {avail }}=E_{\mathrm{txfr}}-\left(E_{\text {diss }}-E_{\text {int }}\right) \text {. }
$$

Since there is insufficient energy available from the collision to generate NO vibrational excitation, the energy available to the $\mathrm{NO}+\mathrm{O}$ fragments $\left(E_{\text {avail }}\right)$ is the sum of the $\mathrm{NO}$ rotational energy, $E_{\mathrm{rot}}$, and the $\mathrm{NO}+\mathrm{O}$ kinetic energy release, $E_{\text {recoil }}$, i.e.,

$$
E_{\text {recoil }}=E_{\text {avail }}-E_{\text {rot }} \text {. }
$$

For a given VMII image of a particular $\mathrm{NO}(J)$ state the rotational energy is fixed. Thus, the distribution of NO recoil energies is determined directly from the distribution of collision energies transferred into the $\mathrm{NO}_{2} * *$ internal degrees of freedom, $E_{\mathrm{txfr}}$, i.e.,

$$
E_{\mathrm{txfr}}=E_{\text {recoil }}+E_{\mathrm{rot}}+\left(E_{\mathrm{diss}}-E_{\mathrm{int}}\right) \text {. }
$$


The experiment measures fragment velocities that are fixed by the energy constraints above as well as the conservation of momentum. While only the NO fragment velocity is measured, this is sufficient to fix the velocity of the $\mathrm{O}$ co-fragment, and thus the $E_{\text {recoil }}$ distribution is uniquely related to the NO recoil velocity distribution. As shown in the $\mathrm{NO}(J)$ velocity schematic of Figure 2, the total NO velocity distribution is a vector sum of this NO recoil velocity $v_{\text {recoil }}$ and the "translational" $\mathrm{NO}$ velocity, $v_{\text {trans, }}$, which is the scattered $\mathrm{NO}_{2} * *$ velocity. If the collision between the $\mathrm{NO}_{2} *$ and $\mathrm{Ar}$ is purely elastic, the $\mathrm{NO}_{2}{ }^{*}$ does not dissociate and the magnitude of the "translational" velocity is determined by the full $E_{\text {coll }}$ value. The conversion of available collision energy, $E_{\text {coll }}$, to internal energy reduces the "translational" velocity by an amount determined by the degree of internal energy excitation, $E_{\mathrm{txfr}}$. This reduction in $v_{\text {trans }}$ is accompanied by a concomitant increase in $v_{\text {recoil }}$, however, this NO recoil velocity is reduced by the degree of rotational excitation in the NO fragment, $E_{\text {rot. }}$ With this full accounting between the measured, known, and unknown quantities of the experiment established, our approach is to image the NO velocity distribution of a range of rotational states and analyze these VMII images through comparison to a model with only one adjustable parameter, the collisional energy transfer distribution $E_{\mathrm{txfr}}$. The shape of the energy transfer function over the energy range any one particular rotational state of the NO is sensitive to will be assumed to be exponential. As each rotational state is sensitive to different regions of excitation energy we will find the best exponential fit for each sub region of energy transferred.

The velocity distribution of the NO CID products is quantified by rotational-stateselective VMII. Images were acquired for a series of energetically-accessible $\mathrm{NO}(J)$ fragments, specifically $J=0.5,1.5,3.5,5.5,6.5$ and 7.5 . The raw images of these rotational quantum states are shown in Figure 3, overlaid by the elastic scattering velocity radius. A few trends are apparent in these images. It is immediately apparent that the clear velocity "rings" of typical inelastic scattering experiments are highly "smeared" by the NO recoil distribution. A clear relationship between the width of the NO velocity distribution and the rotational state is apparent in the images and we investigate the origin of this trend. 

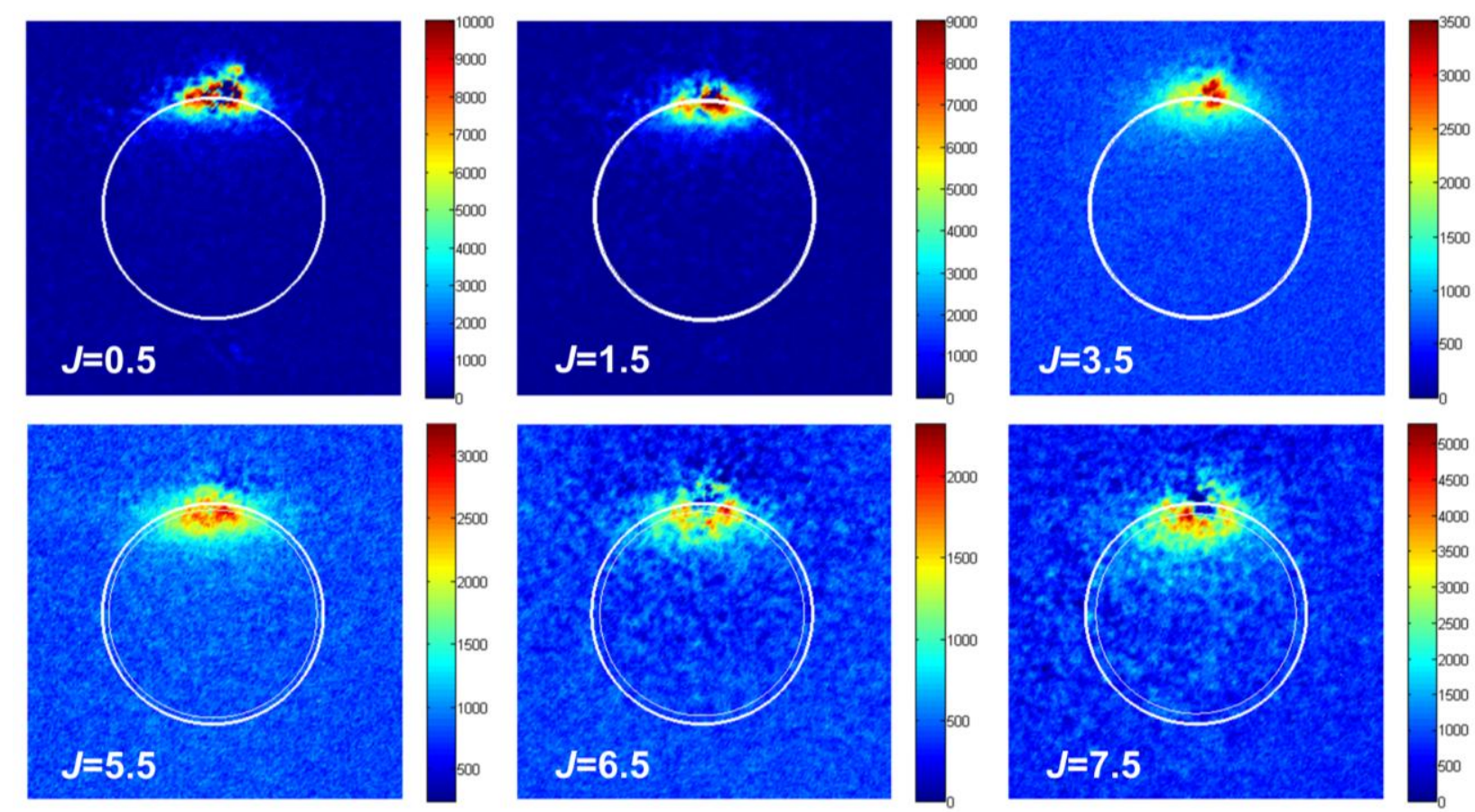

Figure 3. Raw images of velocity distributions from select $\mathrm{NO}(\mathrm{J})$ states. $\mathrm{NO}$ molecules are produced from collisional dissociation resulting from $\mathrm{NO}_{2} *+\mathrm{Ar}$ inelastic scattering. $\mathrm{NO}_{2} *$ forward scattering is shown at the vertical position. The outer white circle shows the elastic scattering radius for $\mathrm{NO}_{2}$ and the inner white circle shows the reduction of velocity equivalent to the degree of energy required to excite the given $\mathrm{NO}(\mathrm{J})$ rotational state from the $\mathrm{NO}+\mathrm{O}$ dissociation threshold.

Due to the increasing degeneracy of the $\mathrm{NO}(J)$ states with energy, an $\mathrm{NO}_{2}{ }^{* *}$ molecule with a certain energy above the dissociation threshold is more likely to produce an energetically accessible high- $J$ state than a low- $J$ state. Ionization from individual $\mathrm{NO}(J)$ rotational states thus produce energy-dependent sensitivities which must be factored into the analysis. These propensities have been previously determined by measuring the product distribution following laser excitation to many different energy above the dissociation limit, ${ }^{65}$ and it has been verified that these follow statistical behavior, ${ }^{78}$ as illustrated in Figure 4. We undertook an experimental reexamination of the $J$-state yield as a function of energy produced by direct single-photon photodissociation of $\mathrm{NO}_{2}$. Measurements employing both fixed-probe scanned pump and fixed pump scanned probe (REMPI spectra) were performed and we confirmed the $\mathrm{NO}(\mathrm{J})$ distributions as a function of energy follow a simple statistical model. The details of this investigation are summarized in Fig. S1 of the Supplementary Information, and our primary conclusion is that we are able to predict the particular and unique $\mathrm{NO}(J)$ distribution that results from a specific above- 


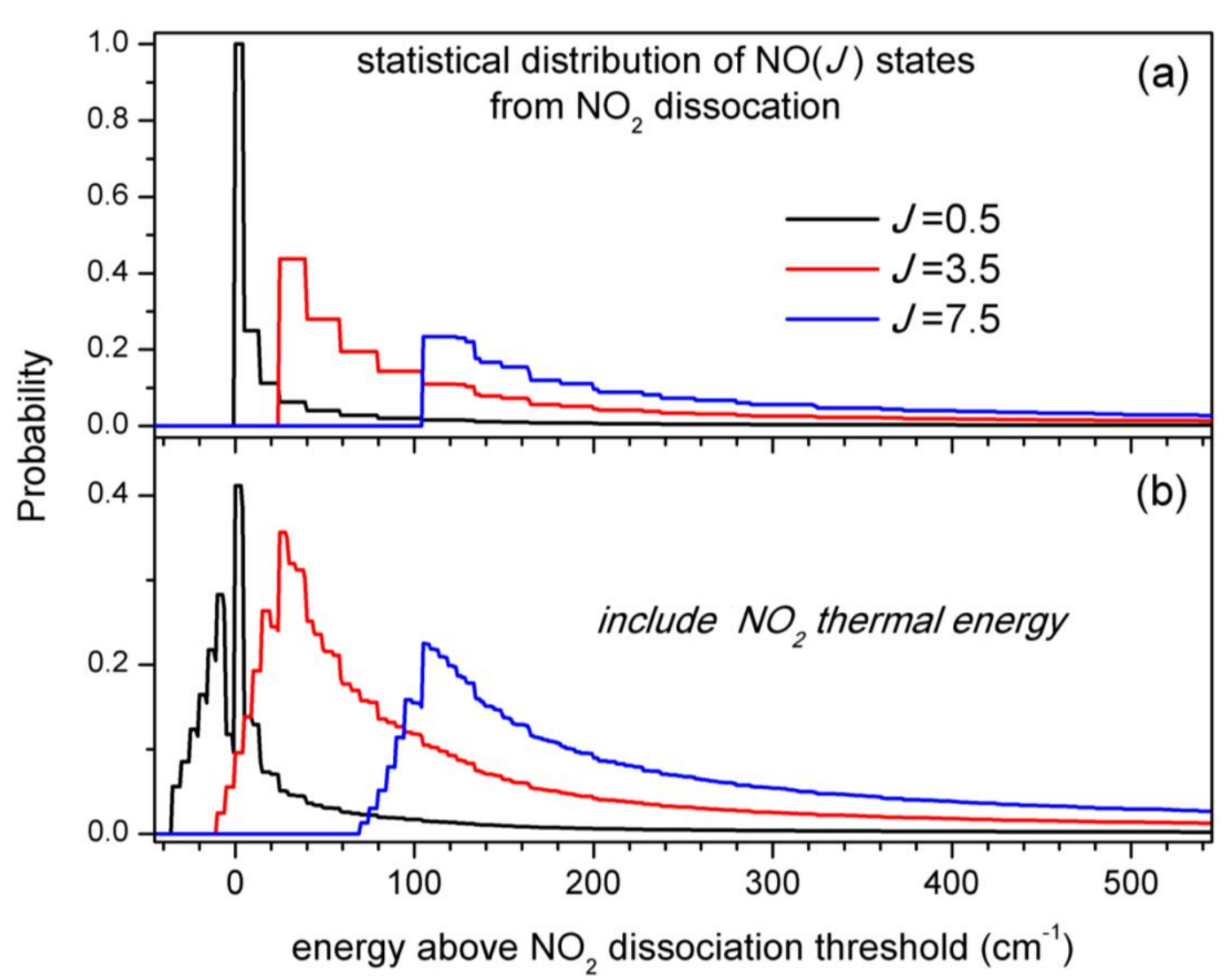

Figure 4. Energy dependence of $\mathrm{NO}(J)$ distributions. The distributions shown in panel (a) follow a statistical model based upon the $(2 J+1)$ rotational state degeneracies, $N O(X)$ spin-orbit excited states, and available $\mathrm{O}\left({ }^{3} \mathrm{P}\right)$ states. These state probabilities show the sensitivity of a given $\mathrm{NO}(J)$ image to a particular degree of collisional energy excitation, and thus each J-state rotationally and vibraitonally excited). Based upon our knowledge of the $\mathrm{NO}(J)$ distribution as a function of energy, we can determine the energy "sensitivity" of a particular $J$-state image in revealing the distribution of collisionally excited energies above $\mathrm{NO}_{2}$ dissociation threshold. As shown in the upper panel of Figure 4, these $\operatorname{NO}(J)$ "windows" dictate how sensitive our individual $J$-state VMII images are to various energies in the dissociating $\mathrm{NO}_{2} * *$ molecule. As illustrated by the lower panel of Figure 4, due to the small degree of internal energy in our $\mathrm{NO}_{2}$ beam, the yield of a given $J$-state image will also include the initial distribution of internal energies. This is because the measured collisional excitation distribution from each $\mathrm{NO}(J)$ state is convolved with the initial thermal energy distribution in the molecular beam. 

excitation distribution from each $\mathrm{NO}(J)$ state is convolved with the initial thermal energy distribution in the molecular beam, as shown in panel (b).

With the particular $\mathrm{NO}(J)$ energy dependent sensitivities in hand we can generate a model of NO $J$-state recoil velocity distribution that is a function of only one parameter, the best exponential shaped collisional energy transfer distribution for each NO rotational state window. Our approach is to compare the model to our experimental data in order to iteratively input the best energy transfer distribution that fits the data. Since we wish to determine the energy transfer distribution with as few assumptions as possible, a model of $\mathrm{NO}$ recoil as a function of $\mathrm{NO}_{2}$ energy is made that eliminates the effect of the $\mathrm{NO}_{2}+$ Ar scattering differential cross-section (DCS). We employ a projection of each of the modeled and observed NO velocity images that describe identical quantities. Specifically, the integration of the signal in the images at a given radius, centered at the center-of-mass of the elastic scattering of the $\mathrm{NO}_{2}+$ Ar collision, around all angles generates a radial distribution function that is sensitive to the energy transfer function and insensitive to the $\mathrm{NO}_{2}+\operatorname{Ar}$ DCS.

The generation of the NO velocity model images is illustrated in Fig. 5. Using the energy- and momentum-conservation principles outlined above, we make a lowered-dimensional model of all the possible $\mathrm{NO}(J)$ velocities that can result from $\mathrm{NO}_{2}{ }^{*}+$ Ar scattering. We assume an isotropic emanation of the NO fragment, thus there is no correlation between the direction of the scattered $\mathrm{NO}_{2} * *$ (the NO $v_{\text {trans }}$ ) and the direction of recoil of the NO fragment ( $\left.v_{\text {recoil }}\right)$, only a correlation of magnitudes. We will show below that breakdown of this assumption may be critical to explaining the discrepancy we will demonstrate between the experimental measurement of the $P\left(E, E^{\prime}\right)$ distribution and the calculated distribution. As shown in panel (a) of Fig. 5, we represent the $\mathrm{NO}$ recoil as two-dimensional circles and the $\mathrm{NO}_{2} * *$ scattering vector as a one-dimensional line. This reduction in dimensionality is properly accounted for in our radial distribution projection analysis, because it preserves cylindrical symmetry about the relative velocity vector of $\mathrm{NO}_{2} *$ and $\mathrm{Ar}$. The importance of this symmetry and the projection of the model and experimental images to an equivalent radial intensity distribution are described in further detail below.

The degree of loss of kinetic energy in the $\mathrm{NO}_{2} *+$ Ar system and the selection of the particular $\mathrm{NO}(J)$ state directly determines the $\mathrm{NO}$ recoil velocity magnitude. As shown by the 
PES schematic in Fig. 5, at each particular collisional $E_{\mathrm{txfr}}$ value (and for each individual $\mathrm{NO}(J)$ state) there is a corresponding "ring" of NO velocities which is a vector sum of the 2-D $v_{\text {recoil }}$ (red circles) and 1-D v $v_{\text {trans }}$ (red 'x's). The sum of all of these possible velocities produces the example NO $J=3.5$ distribution map in the top right (panel (b)) of Fig. 5. Finally, the model is convolved with the available energy distributions; the initial degree of thermal energy in the $\mathrm{NO}_{2}$ beam as well as the $\mathrm{NO}_{2}$ and $\mathrm{Ar}$ beam velocity spreads must be accounted for in terms of the contribution to total available energy. The image in panel (c) of Fig. 5 is the result of the one-dimensional model convolved with the distribution of total available energies. Inherent in this image is that all energies of the $\mathrm{NO}_{2}$ are equally excited (the $P\left(E, E^{\prime}\right)$ distribution is given as a constant with energy), and the $J=3.5$ rotational quantum state detects each of these energies with equal sensitivity (as if at all $\mathrm{NO}_{2}$ energies above the energy of $\mathrm{NO}(J=3.5)$ the $\mathrm{NO}_{2}$ dissociates and only produces $\mathrm{NO}(J=3.5)$. We will add the energy sensitivity window, as is given by the windows discussed above, and fit a energy transfer function to each window to find the best fit to the data.

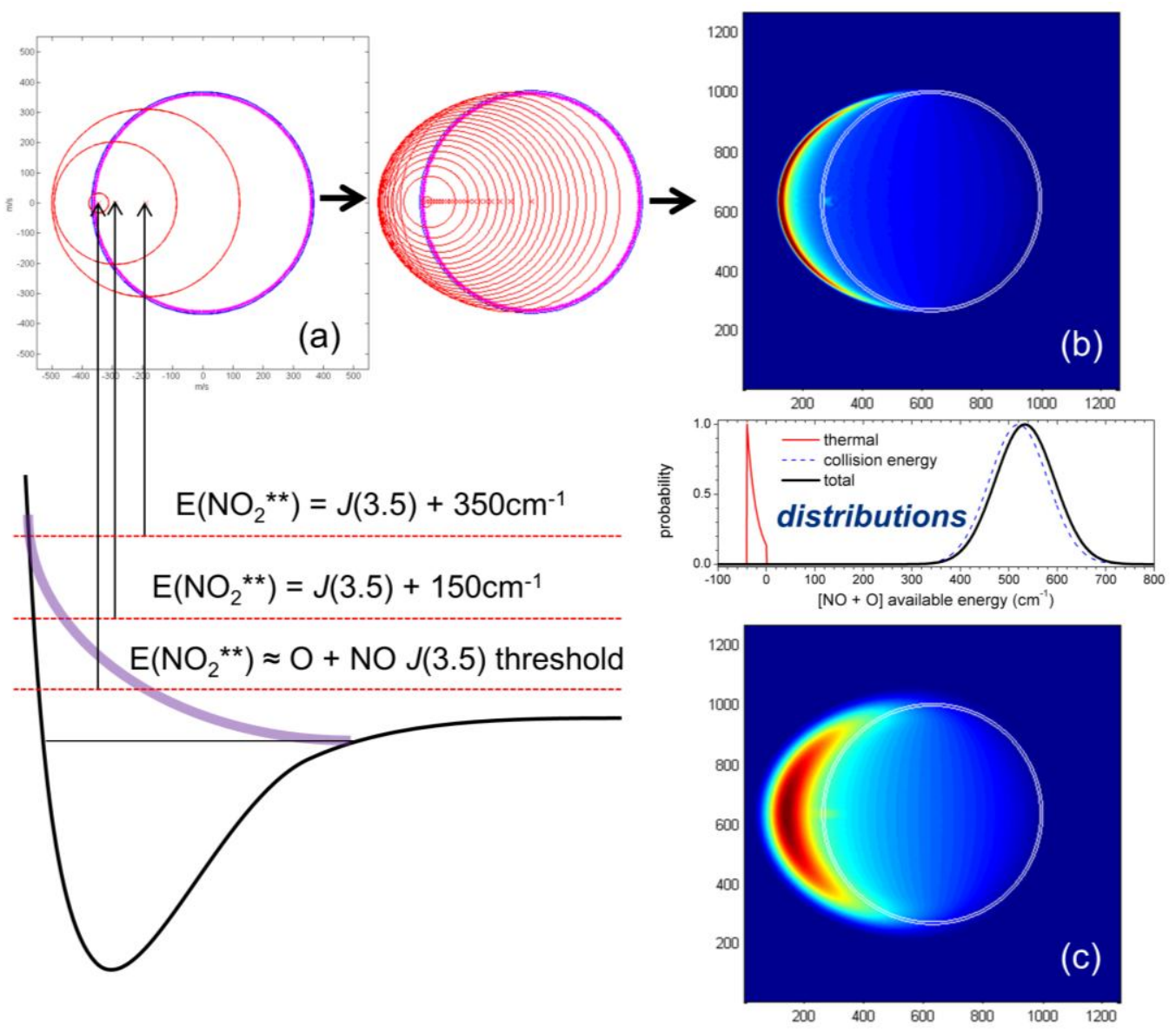


Figure 5. Construction of $J$-state specific NO velocity distribution model for example of $J=3.5$. As shown in panel (a), the increase in $\mathrm{NO}_{2}$ internal energy by reduction of total $\left[\mathrm{NO}_{2}+\mathrm{Ar}\right]$ kinetic energy results in a fixed $[\mathrm{NO}+\mathrm{O}]$ kinetic energy for a particular $\mathrm{NO}(J)$ state. The collection of all possible NO recoil velocities in panel (b) is "smeared" by the available energy distributions; the thermal $\mathrm{NO}_{2}$ in the molecular beam and the beam velocity spreads result in a distribution of total available energies in the system; the resulting distribution of all possible velocities available to the $\mathrm{NO}(J)$ fragment is shown in panel (c).

The distribution shown in Fig. 5c assumes all energetically possible energies are equally populated and the particular $J$ state, $J=3.5$, is equally sensitive to all energies. However, for each NO $J$-state there is a unique sensitivity to the various excess energies of $\mathrm{NO}_{2} * *$ (i.e. the red ' $\mathrm{x}$ 's of Fig. 5 receive different sampling weights for each $J$-state "window"). This involves both an energetic cut-off, as well as a decay in sensitivity with increasing energy as other higher- $J$ "windows" become energetically accessible and "steal" population into these higher rotational states. As described above, these windows are calculated from a statistical account of the state degeneracies. To account for the observed velocity distribution of a particular $N O(J)$ state, this statistical weighting to the energy window of a particular $J$ state must be added to the model of Fig. 5. The results are shown in Fig. 6. Shown as an example in the upper left and right panels of Fig. 6 are the $J(1.5)$ and $J(7.5)$ energy "windows", respectively. The results of the weighting they impose upon all possible NO recoil velocities (i.e. the image in panel (c) of Fig. 5) are shown in the middle velocity images of the figure. These images assumes all possible energies are equally likely, $\mathrm{P}\left(\mathrm{E}, \mathrm{E}^{\prime}\right)$ is a constant, and show only the $\mathrm{NO} J=1.5$ and $J=7.5$ energy sensitivity of the $E_{\mathrm{txfr}}$ measurement. It is clear from the differences between these velocity distribution maps that the NO $J$-state "windows" have a strong effect on what $\mathrm{NO}_{2} * *$ energies are probed. 

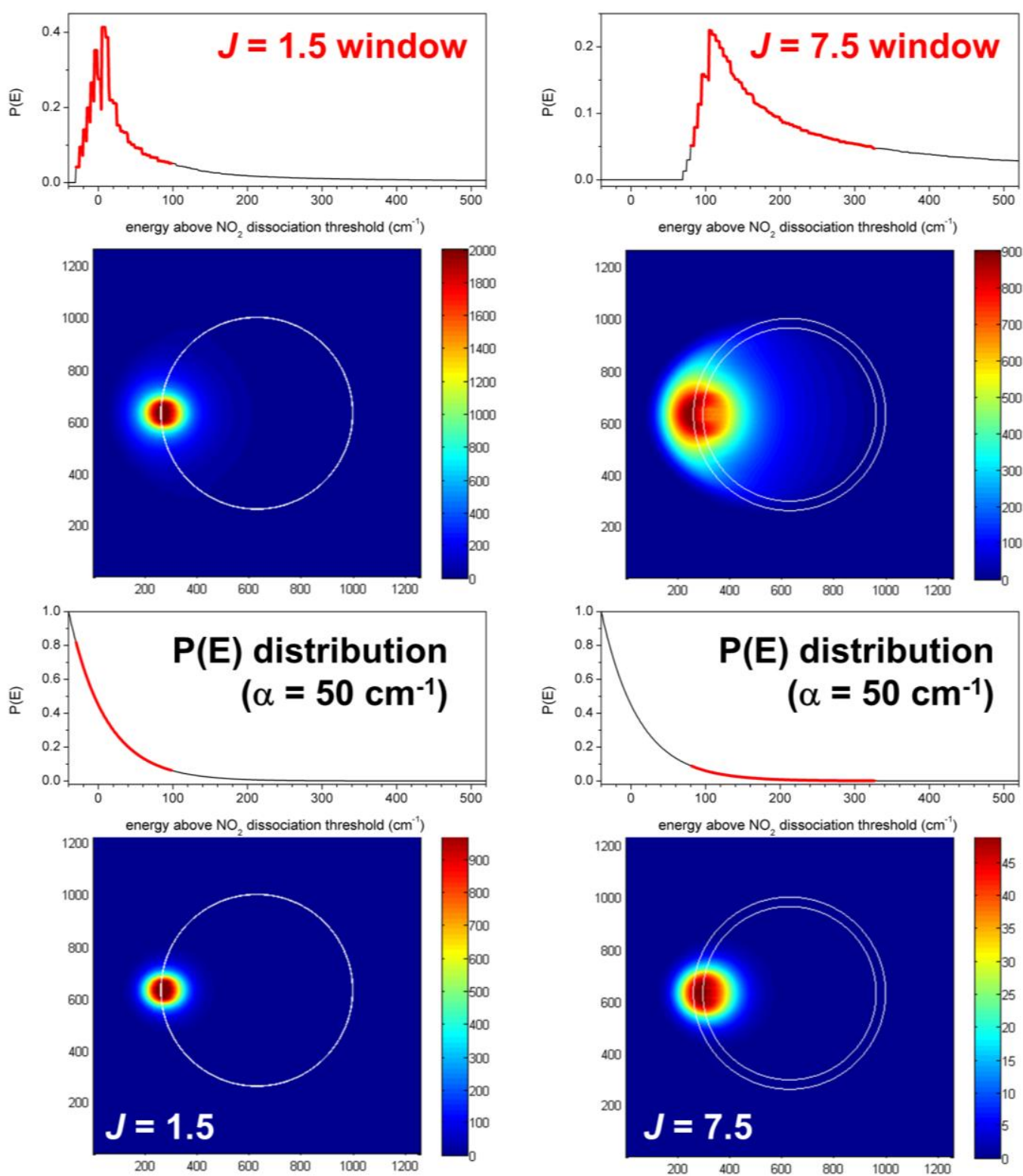
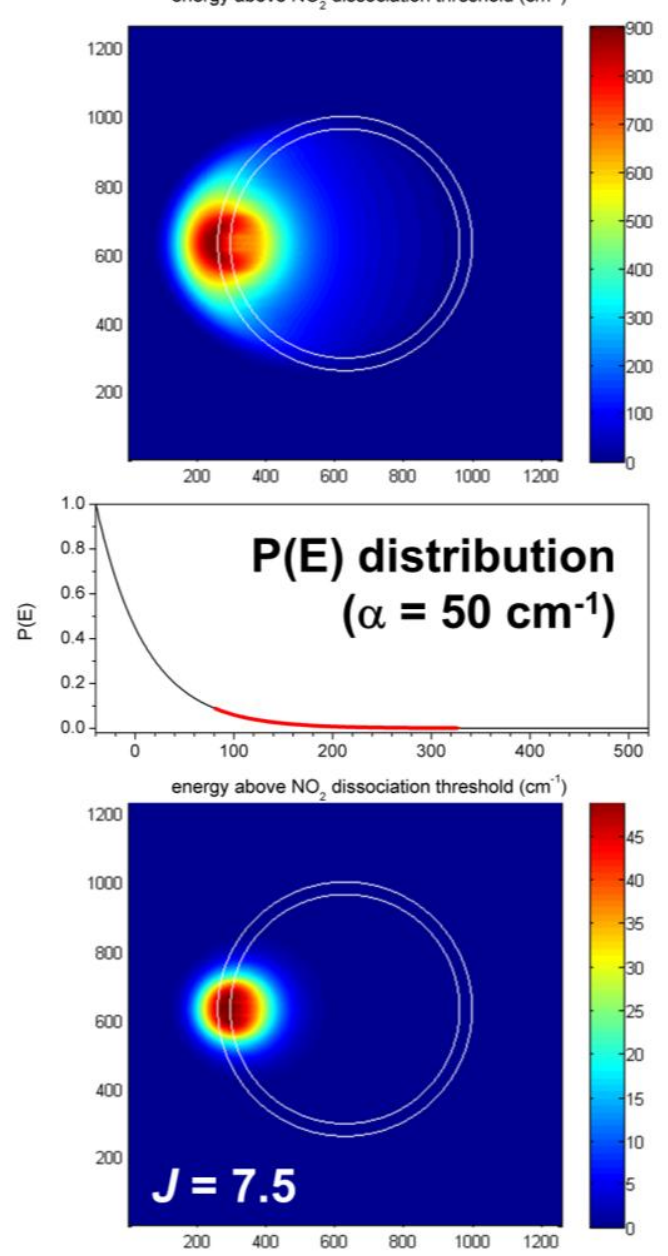

Figure 6. Illustration of $J$-specific sensitivity "windows" and $\mathrm{P}(\mathrm{E})$ energy transfer function weighting on modeled velocity distributions. The $J$-window function dictates the "instrument function" of a particular $\mathrm{NO}(J)$ state towards all the possible $\mathrm{NO}_{2}$ internal energies and mitigates the importance of various NO recoil velocity groups. The extents corresponding to $75 \%$ of total sensitivity are shown by the red overlay on the curves. The $P\left(E, E^{\prime}\right)$ distribution determines the distribution of $\mathrm{NO}_{2}$ energies that produce $\mathrm{NO}$ fragments, and is represented here by a singleexponential decay function. This single-exponential $P\left(E, E^{\prime}\right)$ function is adjusted to fit the data for each $\mathrm{NO}(J)$ image.

The importance of each particular $E_{\mathrm{txfr}}$ value (and thus the $J$-dependent signal from the correlated NO recoil velocity distribution) is given by the collisional energy transfer distribution, 

input and the resulting weighting of the $J$-state model images is calculated. In this case, the trial function of

$$
P\left(E, E^{\prime}\right)=\exp (-\Delta E / \alpha)
$$

where $\alpha=50 \mathrm{~cm}^{-1}$ is shown for both $J$-states. The energy range of the $P\left(E, E^{\prime}\right)$ function to which the particular $J$-state image is sensitive to is shown as a thick red overlay on the $P\left(E, E^{\prime}\right)$ curve. To preserve the straightforward nature of our approach, we model the $P\left(E, E^{\prime}\right)$ function for each $J$-state image as a single exponential decay function, and thus fit the model to the data with a single numerical parameter. This decay constant, $\alpha$, represents the single adjustable parameter of our model, and it is clear from the images in the lower panels of Fig. 6 that there is a strong effect on the modeled NO velocity distribution from introducing this $P\left(E, E^{\prime}\right)$ weighting.

As stated previously, a comparison between the experimental and modeled velocity distributions requires collapsing the images into an image projection that the two share in common. One such projection is the radial distribution of the angle-integrated signal about the center of mass of the $\mathrm{NO}_{2}^{*}+\mathrm{Ar}$ system (as shown by $v_{\mathrm{COM}}$ in Fig. 1(c)). This annular integration allows the effect of the $\mathrm{NO}_{2}{ }^{*}+$ Ar DCS to be ignored and represented by the 1-D model of $\mathrm{NO}_{2}{ }^{* *}$ scattering. (An example justification of this is shown in Fig. S2 of the Supporting Information.) In order to compare with the experimental data, an image transform, inverse Abel transform, is required before angle-integration and comparison to the model because the experimental image represents a 3-D velocity distribution "crushed" onto a 2-D surface. Our images have cylindrical symmetry about the relative $\mathrm{NO}_{2} *+$ Ar velocity vector. For images with this symmetry property, this problem has been previously investigated in detail and we employ the BASEX program of Reisler and co-workers ${ }^{79}$ to perform this inverse Abeltransform. This transform essentially outputs the 2-D basis of the 3-D image, and thus the radial distribution of the image is a direct representation of the magnitudes of the velocity distribution (i.e. NO speeds). The "raw" experimental images are inverse-Abel transformed and integrated annularly to produce an experimental radial distribution that can be directly compared to the simulated radial distribution of the model, these are shown in Fig. 7.

In Fig. 7 is a direct comparison of the radial velocity distribution of the data and the model for each $J$-state for different $\alpha$ parameters for the $P\left(E, E^{\prime}\right)$ trial input. The comparison between data and experiment is used as a basis for an error minimization routine that yields the 

energy "window" for production of a particular $\mathrm{NO} J$-state from $\mathrm{NO}_{2} * *$ has a defined range, this essentially gives the average slope of the $\log \left(P\left(E, E^{\prime}\right)\right)$ function over this energy range. The best single-exponential fit to each $J$-state velocity map image is not the same, and follows a rough increasing trend with energy. The change in the best-fit $\alpha$ value means that the measured collisional energy transfer distribution is best described by a multi-exponential decay.
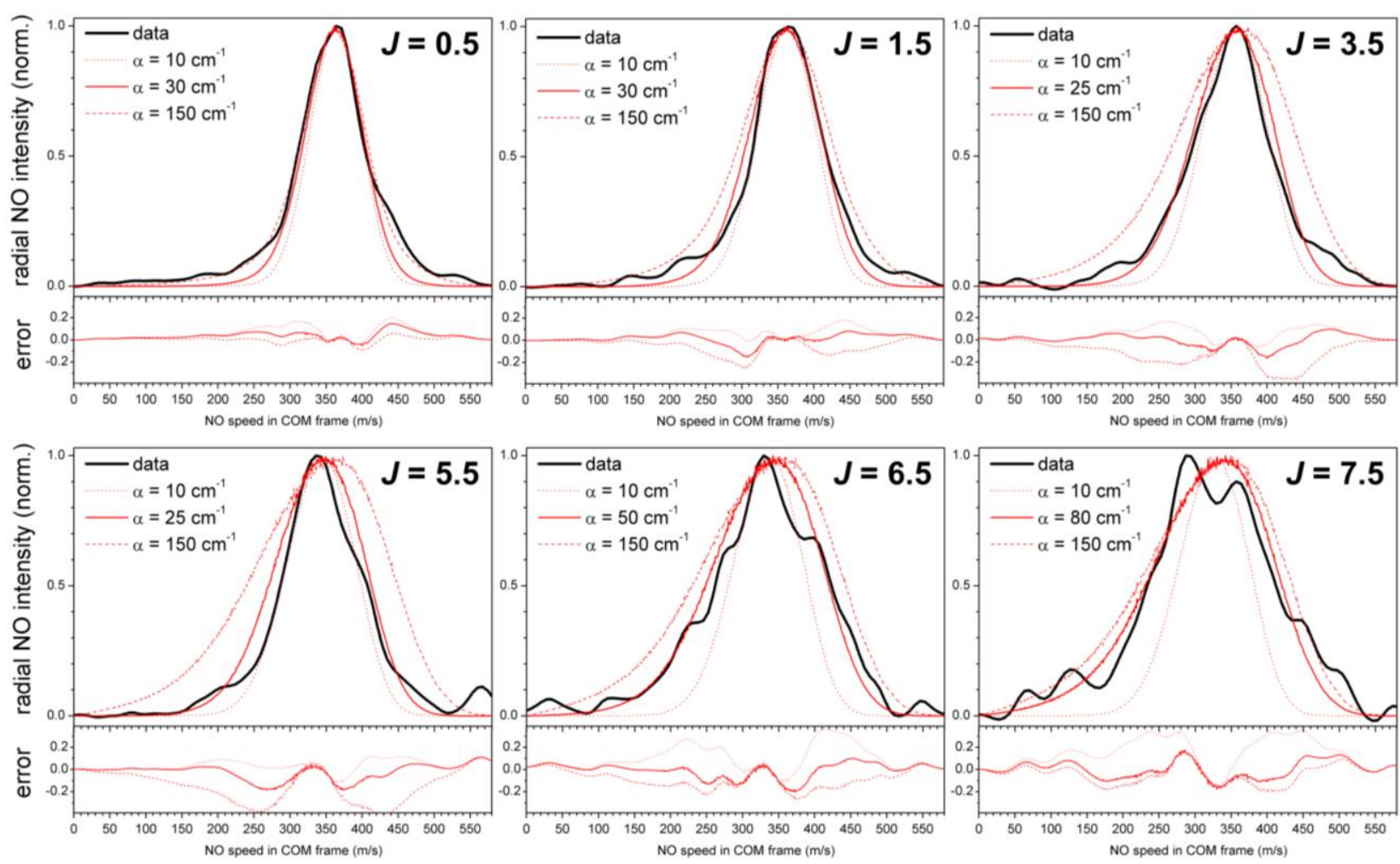

Figure 7. The radial intensity distribution of the NO signal is inverse-Abel transformed and integrated around all scattering angles in order to eliminate the effect of the $\mathrm{NO}_{2}+$ Ar DCS from the analysis. A comparison of the radial intensity distribution from the experimental images to that of the NO recoil model is shown for all the studied $\mathrm{NO}(J)$ states. The comparison shows the best fit single-exponential $P\left(E, E^{\prime}\right)$ function is shown for each $\mathrm{J}$-state, as well as a consistent set of lower and upper bound $P\left(E, E^{\prime}\right)$ trial functions. The error of model vs. data is shown in the lower panels.

The overall behavior of the experimental $P\left(E, E^{\prime}\right)$ distribution over the energy range investigated is summarized in the lower panel of Fig 8. The best-fit single-exponential parameter is plotted vs. the position and extent of the energy "window" each $\mathrm{NO}(J)$ state provides into the $\Delta E\left(E_{\mathrm{txfr}}\right) \mathrm{NO}_{2} * *$ excitation. These best-fit single-exponential decay parameters for each of the $J$-states are $\alpha=34,30,22,27,52$, and $80 \mathrm{~cm}^{-1}$ for $J=0.5,1.5,3.5,5.5,6.5$ and 7.5 , respectively. 


\begin{abstract}
Also shown is the single-exponential fit $\left(\alpha=125 \mathrm{~cm}^{-1}\right)$ of the study of Sanov et al. ${ }^{37}$ who considered a wider and higher energy range. Shown in the upper panel of Fig. 8 are the results of the trajectory calculations over the experimentally-relevant energy range. The multiexponential behavior of the calculated $P\left(E, E^{\prime}\right)$ distribution is immediately apparent. A comparison to the experimental data shows obvious qualitative agreement and rough quantitative agreement. The best single-exponential representation of the calculated $P\left(E, E^{\prime}\right)$ curve over the energy region interrogated by the low- $J$ images is with a decay parameter $\alpha=20 \mathrm{~cm}^{-1}$, which agrees well with the experiment. The approximate single-exponential representation of $P\left(E, E^{\prime}\right)$ over the higher- $J$ state energy region of the experiment is in the range of $100-300 \mathrm{~cm}^{-1}$, in rough agreement with the current study and the previous work of Sanov et al. ${ }^{37}$
\end{abstract}




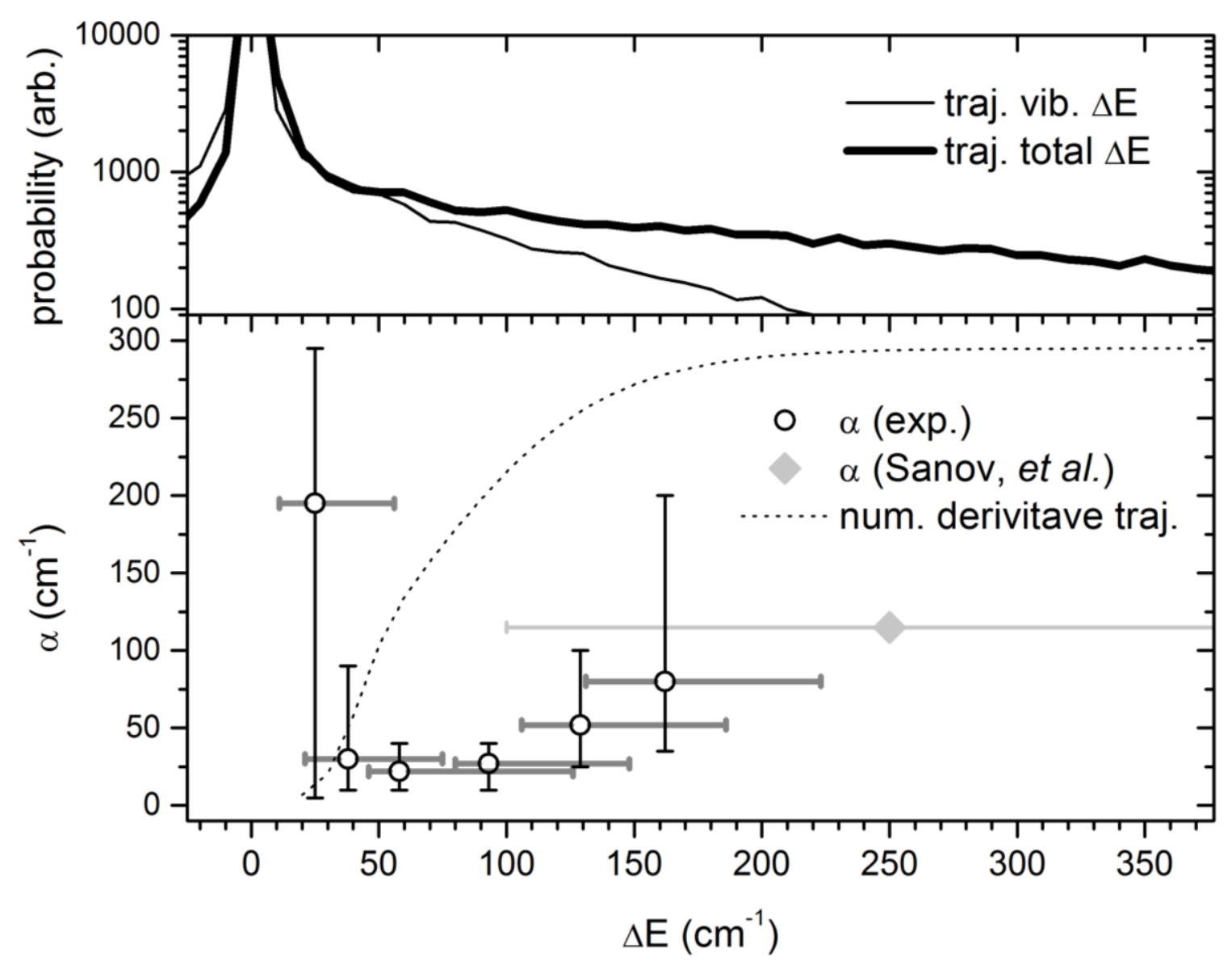

Figure 8. A comparison of experimentally-determined and calculated $P\left(E, E^{\prime}\right)$ distributions. The trajectory $P\left(E, E^{\prime}\right)$ results are shown in the in the upper panel, and the numerical derivative of this distribution is comparable to the single-exponential fits, and shown as a dotted line in the lower panlel. The best fit single-exponential $P\left(E, E^{\prime}\right)$ function is shown in the lower panel for each $J$ state as the vertical position of a horizontal line. The open circle shows the "center-of-mass" of the $J$-state's "window", and the length of the line corresponds to the extent of that particular $J$ state "window" used to interrogate the $\mathrm{NO}_{2} P(E, E$ '). Also shown is the experimental result from a previous experimental study of Sanov et al., with the corresponding energy range that was interrogated. The current study clearly shows a multi-exponential $P\left(E, E^{\prime}\right)$ shape, in qualitative agreement with the calculations.

Despite the qualitative agreement between the experimental result for the $P\left(E, E^{\prime}\right)$ behavior and the QCT trajectory results, there is clear quantitative discrepancy. The experimental approach is to report a best-fit characterization of the real $P\left(E, E^{\prime}\right)$ over the energy sensitivity of a particular $\mathrm{NO}(\mathrm{J})$ image. As a check on comparison between the trajectory calculations and the experiment we have taken the $P\left(E, E^{\prime}\right)$ as determined by the QCT and predicted the velocity 

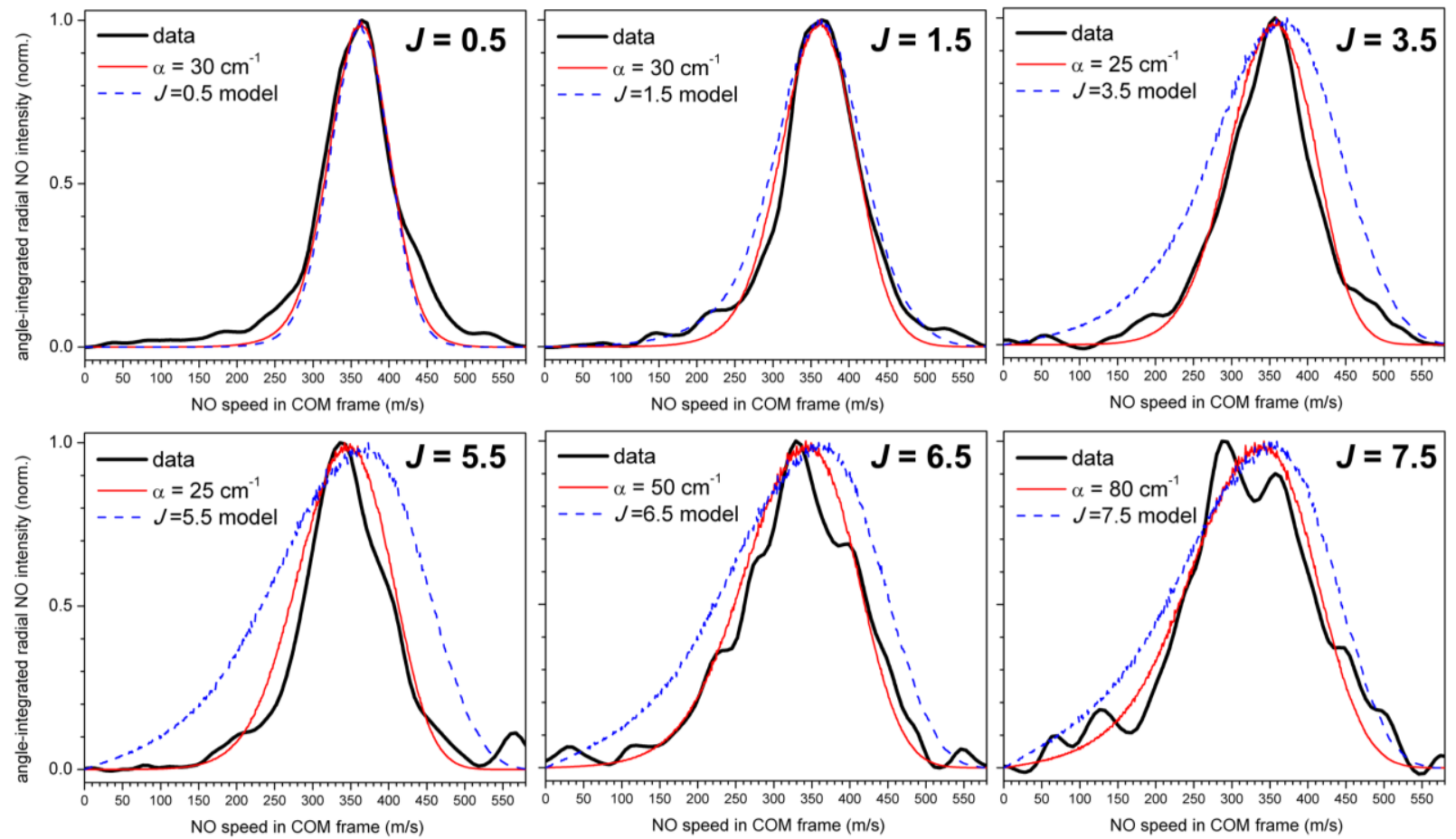

Figure 9. A comparison of the experimental and modeled radial intensity distributions using the QCT P(E) for each the studied $\mathrm{NO}(\mathrm{J})$ states. The comparison shows the data, the best fit singleexponential $\mathrm{P}(\mathrm{E})$ function, and the the result of the $\mathrm{P}(\mathrm{E})_{\mathrm{QCT}}$.

A variety of sources were considered for the quantitative disagreement between the experimental and calculated results. There are assumptions in both the trajectory calculations and 
experimental analysis that may lead to this discrepancy. The possibility exists that the experiment is biased towards the detection of certain types of energy transfer, in that there is a fundamental assumption of statistical dissociation dynamics from $\mathrm{NO}_{2} * *$ to make the distribution of $\mathrm{NO}(\mathrm{J})$ states. While we verified the statistical distribution of $\mathrm{NO}(\mathrm{J})$ states from $\mathrm{NO}_{2} * *$ molecules prepared by direct photodissociation, we do not directly do so for the $\mathrm{NO}(\mathrm{J})$ distribution produced from CID, but rather assume the statistical behavior as an underpinning of our analysis. Another possibility is that the presence of the $\mathrm{NO}_{2}$ electronic excited state may significantly affect the energy transfer process, which was modeled only with the ground state manifold of internal states.

Finally, any effect that created a non-isotropic dissociation of the $\mathrm{NO}_{2} * *$ creating a nonisotropic distribution NO products would affect our analysis and fitting. Two possible effects were investigated that could cause a non-isotropic NO distribution. First, if the collisional excitation of $\mathrm{NO}_{2} *$ to high internal energies is a non-isotropic event and the highly excited $\mathrm{NO}_{2} * *$ fell apart before it had a chance to rotate and randomize its position then the resulting distribution of NO would be non-isotropic. Second, if the assumption that the collision event is temporally distinguishable from the unimolecular dissociation due to the rapidity of the scattering event is incorrect and some fraction of the quickly dissociating $\mathrm{NO}_{2}{ }^{* *}$ molecules produce NO which has a subsequent collision (either elastic but direction changing or inelastic) with the Ar atom that excited the $\mathrm{NO}_{2}{ }^{*}$, then a non-isotropic $\mathrm{NO}$ distribution would be formed.

To investigate the possibility of non-isotropic collisional excitation of $\mathrm{NO}_{2}{ }^{*}$, we analyzed the ensemble of trajectories used to generate figure 8. For each trajectory in the ensemble, the smallest of the two Ar-N-O angles, $\theta_{\mathrm{ArNO}}$, was calculated at the "impact geometry," defined here as the geometry along the trajectory with the shortest $\mathrm{Ar}-\mathrm{NO}_{2}$ center-of-mass distance. Figure 10 shows the fraction collisions that transfer at least $300 \mathrm{~cm}^{-1}$ binned as a function of $\theta_{\mathrm{ArNO}}$. (One could distinguish collisions that occur on the concave side of $\mathrm{NO}_{2}$ from those on the convex side, but we do not do so in figure 10 as this distinction complicates the figure but does not affect the present conclusions.) One can see from figure 10 that low angle collisions, which correspond to Ar striking the $\mathrm{O}$ atom along an $\mathrm{NO}$ axis, are $\sim 5$ times less likely to transfer at least $300 \mathrm{~cm}^{-1}$ than collisions where Ar strikes an NO axis at angles between 25 and $65^{\circ}$. Above $65^{\circ}$ the likelihood of energetic collisions declines, where angles near $130^{\circ}$ correspond to Ar striking $\mathrm{NO}_{2}$ along its $\mathrm{C}_{2 \mathrm{v}}$ axis. Figure 10 thus demonstrates non-isotropic excitation for high-energy 
collisions. Similar distributions may be obtained for cutoffs larger than $300 \mathrm{~cm}^{-1}$, while the distribution necessarily approaches an isotropic one as the cutoff is lowered to include all collision outcomes.

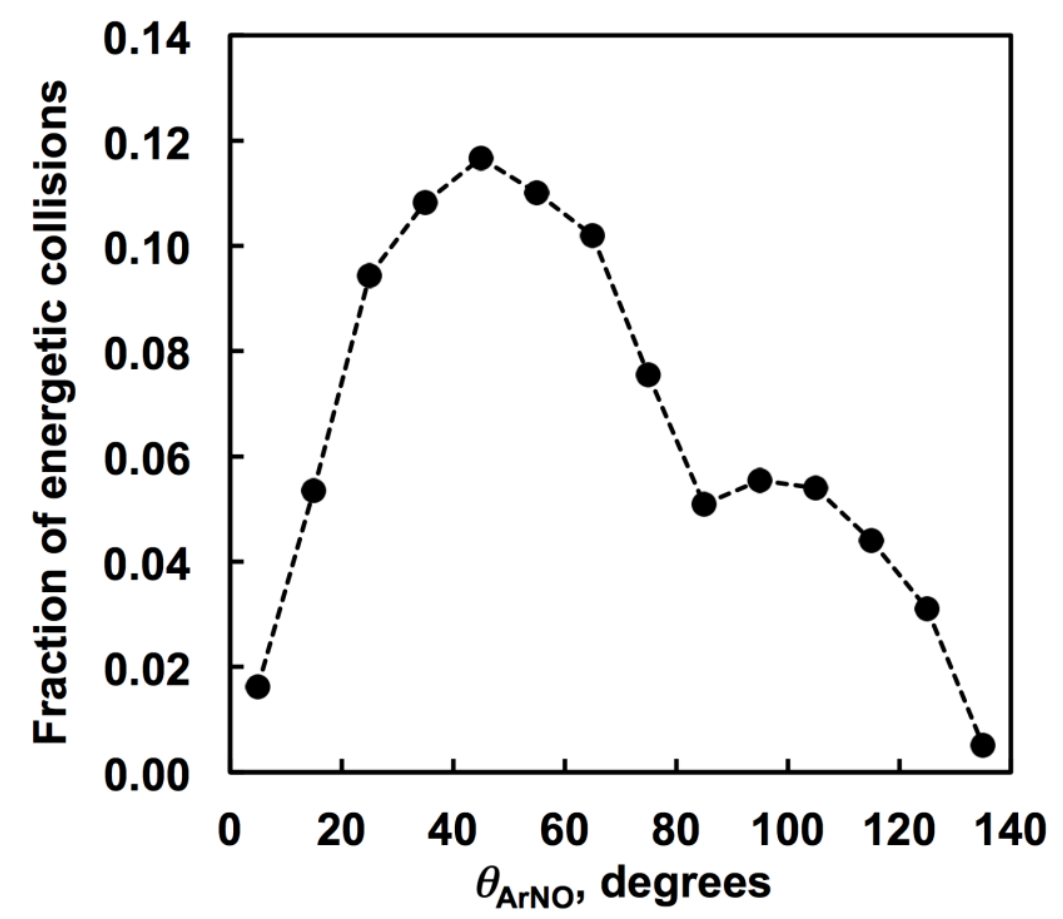

Figure 10. The fraction of collisions that transfer at least $300 \mathrm{~cm}^{-1}$ of energy as a function of $\theta_{\mathrm{ArNO}}$. The results are shown binned with respect to $\theta_{\mathrm{ArNO}}$, with a bin size of $10^{\circ}$.

As the rotational constants of $\mathrm{NO}_{2}$ are small and $\mathrm{NO}_{2}$ is initially rotationally cold, it takes several picoseconds, depending upon the exact rotational excitation, for $\mathrm{NO}_{2}$ to rotate. If the dissociation of $\mathrm{NO}_{2}$ is fast on this time scale, then the angular distribution of the $\mathrm{NO}$ product fragment may reflect this excitation anisotropy. The trajectory results indicate that the degree of anisotropy should increase with increasing excitation, which, on average, also results in faster dissociation rates for $\mathrm{NO}_{2}$. The presence of non-isotropic scattering of $\mathrm{NO}$ may therefore have a quantifiable effect on the present analysis, particularly for large $\Delta E$. A second related effect might further complicate the present interpretation. If one assumes that $\mathrm{NO}_{2}$ dissociation along the center-of-mass vector of $\mathrm{O}-\mathrm{NO}$ vector is fast enough to occur while $\mathrm{Ar}$ is still interacting with $\mathrm{NO}_{2}$, it is instructive to correlate the angle of the departing $\mathrm{NO}$ fragment with $\theta_{\mathrm{ArNO}}$. On average, the distance of closest $\mathrm{Ar}-\mathrm{NO}_{2}$ approach is $\sim 3 \AA$, and using this distance one calculates 
that the departing NO fragment would be directed toward $\mathrm{Ar}$ for $\theta_{\mathrm{ArNO}} \approx 35^{\circ}$. Notably, this value of $\theta_{\mathrm{ArNO}}$ is close to the peak in the distribution of energetic collisions shown in figure 10 . Assuming fast dissociation, energetic collisions are therefore more likely to dissociate in such a way as to interact with Ar. Therefore it is quite reasonable that some fraction of the dissociating NO molecules will feel the presence of the Ar atom either while dissociating or with as a subsequent collision after dissociation, particularly for energetic collisions as these collisions necessarily result in slowly moving Ar atoms. Such events may be observed via classical simulations, but we do not attempt to quantify them here as: (1) the present potential energy surfaces do not include reliable $\mathrm{Ar}-\mathrm{O}$ or $\mathrm{Ar}-\mathrm{NO}$ interactions, and (2) classical mechanics is likely not suitable for modeling such threshold dissociation events. However, due to the fast dissociation rates reported elsewhere ${ }^{80}$ for $\mathrm{NO}_{2}{ }^{* *}$, interactions may be possible such that the kinetic energies of the $\mathrm{NO}+\mathrm{O}$ dissociative recoil are perturbed by the Ar collider. If the present reaction were considered in the reverse direction, such a situation may be considered a "three body" collision. Both of the effects identified here would have the result of diminishing the most forward scattered experimental trajectories thereby narrowing the experimental velocity distribution of Figures 7 and 9 making it appear that less energy has been transferred by the Ar$\mathrm{NO}_{2}$ collision. Neither of these velocity changing mechanisms would effect the results of the Reisler group as they did not extract the $P\left(E, E^{\prime}\right)$ curve from the velocity distribution of the NO fragment.

Fundamentally, the analysis in Fig. 7 and the comparison in Fig. 9 demonstrates that the experimental data is certainly sensitive to the details of the $P\left(E, E^{\prime}\right)$ and that the $P\left(E, E^{\prime}\right)$ manifests distinctly multi-exponential behavior. In general, it is worth noting that the QCT calculations and the experimental results do agree in the rough slope of the $P\left(E, E^{\prime}\right)$ function at the most likely events, those of low energy transfer, and these show an approximate behavior of $P\left(E, E^{\prime}\right)=\exp (-$ $\left.\Delta E / 20 \mathrm{~cm}^{-1}\right)$.

While the degree of curvature of the $P\left(E, E^{\prime}\right)$ curve remains open to question, the overall behavior of the QCT trajectories is representative, and thus there are some interesting implications in the overall shape of the QCT $P\left(E, E^{\prime}\right)$ curve that we note. Two traces are shown for the trajectory results in Fig. 8; one represents the total energy transfer in terms of internal energy of $\mathrm{NO}_{2}{ }^{*}$ and the other represents only the vibrational component of this energy transfer. We note the first moment of the total energy transfer distribution $(\langle\Delta E\rangle)$ is positive, but that of 
the vibrational energy transfer distribution is negative. We reiterate that these are collisions of a rotationally cold but vibrationally hot molecule, and thus due to the facile interconversion of translational and rotational energies the $\mathrm{NO}_{2} *$ molecules are, on average, energized by a single collision. Of course, the rotational heating is opposite the change in vibrational energy and the trend towards equilibration of the modes is consistent. However, it is worth noting the clear qualitative differences between the first collision(s) of the molecule and later collisions, where rotational and vibrational "temperatures" are similar. This emphasizes the advantage of an approach that does not average over many collisions to determine collisional energy transfer characteristics. Another detail that is clear in the trajectory calculation results is despite the overall dominance of rotational energy transfer for $\Delta E_{\text {up }}$ collisions, at low $\Delta E_{\text {up }}$ values $\left(<60 \mathrm{~cm}^{-}\right.$ ${ }^{1}$ ) the energy transfer is almost entirely due to further vibrational excitation. This may be related to the longer-range interaction for vibrational excitation and be highly sensitive to the collision partner.

\section{Conclusions}

Measurements of NO velocities from collisional dissociation provide sufficient information to determine the $P\left(E, E^{\prime}\right)$ distribution from $\mathrm{NO}_{2}{ }^{*}+\mathrm{Ar}$ collisions under some simplifying assumptions about the dissociation of the $\mathrm{NO}_{2}$ into $\mathrm{NO}+\mathrm{O}$. A model was successfully developed to analyze the $J$-state specific NO velocity distributions. A multiexponential energy transfer distribution has been definitively measured for energy-upward collisions for the first time. This measurement qualitatively agrees with the predictions from theoretical calculations and previous experiments but not quantitatively. One very possible reason for this is that the dynamics of the $\mathrm{NO}_{2}$ dissociation is non-isotropic and affected by the presence of the Ar atom and this would affect the energy transfer distribution we extract from the data. A more complete calculation including excited electronic states may also help bring the calculation into better agreement with the experiments. This particular experimental approach is applicable to other molecular systems with a pair of dissociation fragments where one fragment can be interrogated by rotationally-state-selective REMPI and the other fragment is monatomic, such as acetylene. 


\section{Acknowledgement}

This work is supported by the Division of Chemical Sciences, Geosciences, and Biosciences, Office of Basic Energy Sciences, U.S. Department of Energy. Sandia is a multiprogram laboratory operated by Sandia Corporation, a Lockheed Martin Company, for the United States Department of Energy under Contract No. DE-AC04-94-AL85000. 


\section{References}

1 G. W. Flynn, C. S. Parmenter, A. M. Wodtke, J. Phys. Chem. 100 (1996) 12817-12838.

2 H. Hippler, J. Troe, in Advances in Gas-Phase Photochemistry and Kinetics: Bimolecular Collisions, M. N. Ashford, J. E. Baggot, eds. Royal Society of Chemistry, London, 1989, pp. 206-262.

$3 \quad$ R. E. Weston Jr., G. W. Flynn, Annu. Rev. Phys. Chem. 43 (1992) 559.

4 A. Schiffmann, D. W. Chandler, Int. Rev. Phys. Chem. 14 (1995) 371-420.

5 F. J. Fletcher, B. S. Rabinovitch, K. W. Watkins, and D. J. Locker, J. Phys. Chem. 70 (1966) 2823-2833.

6 J. V. Michel, W. A. Payne, and D. A. Whytock, J. Chem. Phys. 65 (1976) 4830-4834.

7 J. V. Michael, M.-C. Su, J. W. Sutherland, J. J. Carroll, and A. F. Wagner, J. Phys. Chem. 106 (2002) 5297-5313.

8 G. Altinay and R. G. Macdonald, J. Phys. Chem. A 116 (2012) 1353-1367; ibid., 2161-2176.

$9 \quad$ J. R. Barker, J. Phys. Chem. 88 (1984) 11-18.

10 J. Shi and J. R. Barker, J. Chem. Phys. 88 (1998) 6219-6227.

11 G. V. Hartland, D. Qin, H.-L. Dai, J. Chem. Phys. 102 (1995) 8677-8680

12 G. V. Hartland, D. Qin, H.-L. Dai, and C. Chen, J. Chem. Phys. 107 (1997) 2890-2902.

13 H. Hippler, J. Troe, and H. J. Wendelken, J. Chem. Phys. 78 (1983) 6709-6717.

14 J. E. Dove, H. Hippler, and J. Troe, J. Chem. Phys. 82 (1985) 1907-1919.

15 U. Hold, T. Lenzer, K. Luther, K. Reihs, and A. C. Symonds, J. Chem. Phys. 112 (2000)

16 T. Lenzer, K. Luther, K. Reihs, and A. C. Symonds, J. Chem. Phys. 112 (2000) 4090-4110.

17 T. Lenzer and K. Luther, Phys. Chem. Chem. Phys. 6 (2004) 955-959.

18 N. J. Brown and J. A. Miller, J. Chem. Phys. 80 (1984) 5568-5580.

19 C. A. Michaels, Z. Lin, A. S. Mullin, H. C. Tapalian, and G. W. Flynn, J. Chem. Phys. 106 (1997) 7055-7071.

20 J. Du, N. A. Sassin, D. K. Havey, K. Hsu, and A. S. Mullin, J. Phys. Chem. A, ASAP. DOI: $10.1021 / \mathrm{jp} 404939 \mathrm{~s}$

21 L. Yuan, J. Du, and A. S. Mullin, J. Chem. Phys. 129 (2008) 014303.

22 J. A. Johnson, A. M. Duffin, B. J. Hom, K. E. Jackson, and E. T. Sevy, J. Chem. Phys. 128 (2008) 054304. 
23 M. S. Elioff, M. Fang, A. S. Mullin, J. Chem. Phys. 115 (2001) 6990.

24 J. Park, L. Shum, A. S. Lemoff, K. Wener K, and A. S. Mullin, J. Chem. Phys. 117 (2002) 5221.

25 G. C. Schatz and M. A. Ratner, Quantum Mechanics in Chemistry (Prentice Hall, Englewood Cliffs, NJ. 1993).

26 C. Higgins, Q. Ju, N. Seiser, G. W. Flynn, and S. Chapman, J. Phys. Chem. A 105 (2001) 2858-2866.

27 E. Dashevskaya, E. E. Nikitin, and I. Oref, J. Phys. Chem. 97 (1993) 9397.

28 D. L. Clarke, K. C. Thompson, and R. G. Gilbert, Chem. Phys. Lett. 182 (1991) 357-362.

29 A. J. McCaffery, Z. T. Alwhabi, M. A. Osborn, and C. J. Williams, J. Chem. Phys. 98 (2004) 4586-4602.

30 P. L. Houston, R. Conte, and J. M. Bowman, J. Phys. Chem. A 118 (2014) $7758-7775$.

31 D. J. Krajnovich, K. W. Butz, H. Du, and C. S. Parmenter, J. Chem. Phys. 91 (1989) 77057724.

32 D. L. Catlett, Jr., C. S. Parmenter and C. J. Pursell, J. Phys. Chem. 99 (1995) 7371-7380.

33 T. Donohue, M. S. Chou, and G. A. Fisk, Chem .Phys. 2 (1973) 271.

34 F. F. Crim, M. S. Chou, and G. A. Fisk, Chem. Phys. 2 (1973) 285.

35 G. A. Fisk and F. F. Crim, Acc. Chem. Res. 10 (177) 73-79.

36 C. R. Bieler, A. Sanov, M. Hunter and H. Reisler, J. Phys. Chem. 98 (1994) 1058-1060.

37 A. Sanov, C. R. Bieler, and H. Reisler, J. Phys. Chem. 99 (1995) 7339.

38 C. R. Bieler, A. Sanov, C. Capellos and H. Reisler, J. Phys. Chem. 100 (1996) 3882-3887.

39 H. C. Hsu, M.-T. Tsai, Y. A. Dyakov, and C.-K. Ni, Int. Rev. Phys. Chem. 31 (2012) 201233.

40 C. L. Liu, H. C. Hsu, J. J. Lyu, and C. K. Ni, J. Chem. Phys. 124 (2006) 054302.

41 V. Bernshtein, and I. Oref, J. Chem. Phys. 125 (2006) 133105.

42 M. J. Wilhelm, M. Nikow, J. M. Smith, and H.-L. Dai, J. Phys. Chem. Lett. 4 (2013) 23-29.

43 B. Abel, H. H. Hamann, N. Lange, Faraday Discuss. 102 (1995) 147-166.

44 X. C. Hu and W. L. Hase, J. Phys. Chem. 92 (1988) 4040-4046.

45 M. Bruehl and G. C. Schatz, J. Phys. Chem. 92 (1988) 7223-7229.

46 K. F. Lim and R. G. Gilbert, J. Phys. Chem. 94 (1990) 72-77; ibid., 77-84. 
47 G. Lendvay and G. C. Schatz, J. Phys. Chem. 94 (1990) 8864-8866.

R. G. Gilbert, Int. Rev. Phys. Chem. 10 (1991) 319-347.

49 T. Lenzer, K. Luther, J. Troe, R. G. Gilbert, and K. F. Lim, J. Chem. Phys. 103 (1995) 626641.

T. Lenzer and K. Luther, J. Chem. Phys. 105 (1996) 10944-10953.

O. Meroueh and W. L. Hase, J. Phys. Chem. A 103 (1999) 3981.

52 L. M. Yoder and J. R. Barker, J. Phys. Chem. A 104 (2000) 10184-10193.

53 A. L. Brunsvold, D. J. Garton, T. K. Minton, D. Troya, and G. C. Schatz, J. Chem. Phys. $121(2004) 11702$.

54 I. Oref, Isr. J. Chem. 47 (2007) 205-214.

55 J. R. Barker and R. E. Weston, Jr, J. Phys. Chem. A 114 (2010) 10619-10633.

56 M. V. Ivanov and D. Babikov, J. Chem. Phys. 136 (2012) 184304.

57 R. Conte, P. L. Houston, and J. M. Bowman, J. Phys. Chem. A 117 (2013) 14028-14041.

58 R. Conte, P. L. Houston, and J. M. Bowman, J. Phys. Chem. A 118 (2014) 7742-7757.

59 A. W. Jasper and J. A. Miller, J. Phys. Chem. A 113 (2009) 5612-5619.

60 A. W. Jasper and J. A. Miller, J. Phys. Chem. A 115 (2011) 6438-6455.

61 A. W. Jasper, J. A. Miller, and S. J. Klippenstein, J. Phys. Chem. A, 117 (2013) 1224312255.

62 A. W. Jasper, K. M. Pelzer, J. A. Miller, E. Kamarchik, L. B. Harding, and S. J. Klippenstein, Science 346 (2014) 1212-1215.

63 P. Jansen, D. W. Chandler, and K. E. Strecker, Rev. Sci. Instrum. 80 (2009) 083105.

64 I. Bezel, D. Stolyarov, C. Wittig, J. Phys. Chem. A 103 (1999) 10268-10273.

65 U. Robra, H. Zacharias, and K. H. Welge, Z. Phys. D-Atoms, Molecules and Clusters 16 (1990) 175.

66 L. B. Harding, H. Stark, J. Troe, and V. G. Ushakov, Phys. Chem. Chem. Phys. 1 (1999) 6372.

67 D. Reignier, T. Stoecklin, P. Halvick, A. Voronin, and J. C. Rayez, Phys. Chem. Chem. Phys. 3 (2001) 2726.

68 A. J. C. Varandas, J. Chem. Phys. 119 (2003) 2596.

69 M. V. Ivanov, H. Zhu, and R. Schinke, J. Chem. Phys. 126 (2007) 054304. 
70 M. P. Deskevich, D. J. Nesbitt, and H. J. Werner, J. Chem. Phys. 120 (2004) 7281-7289.

71 R. Dawes, A. W. Jasper, C. Tao, C. Richmond, C. Mukarakate, S. H. Kable, and S. A. Reid, J. Phys. Chem. Lett. 1 (2010) 641-646.

72 NIST Chemistry WebBook, NIST Standard Reference Database Number 69, edited by P. J. Linstrom and W. G. Mallard (National Institute of Standards and Technology, Gaithersburg MD, 2013), http://webbook.nist.gov.

73 A. W. Jasper and R. Dawes, J. Chem. Phys. 139 (2013) 154313.

74 X. Hu and W. L. Hase, J. Phys. Chem. 92 (1988) 4040-4064

75 K. F. Lim, J. Chem. Phys. 100 (1994) 7385-7399

76 A. W. Jasper, C. M. Oana, and J. A. Miller, Proc. Combust. Inst. 35 (2015) 197-204.

77 A. W. Jasper, E. Kamarchik, J. A. Miller, and S. J. Klippenstein, J. Chem. Phys. 141 (2014) 124313.

78 J. Miyawaki, K. Yamanouchi, and S. Tsuchiya, J. Chem. Phys. 99 (1993) 254.

79 V. Dribinski, A. Ossadtchi, V. A. Mandelshtam, and H. Reisler, Rev. Sci. Instrum. 73 (2002) 2634-2642.

80 B. Kirmse, B. Abel, D. Schwarzer, S. Y. Grebenshchikov, and R. Schinke, J. Phys. Chem. A 104 (2000) 10398-10408. 


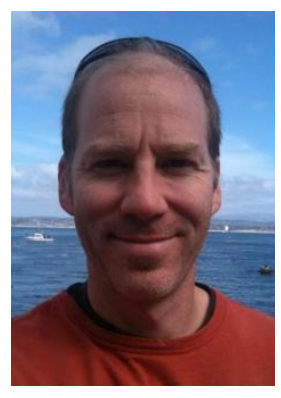

Jeffrey D. Steill received his B.S. in Chemistry and then Ph.D. in Chemical Physics from The University of Tennessee in 2007 with Prof. Robert N. Compton. He continued research in ion spectroscopy in a postdoctoral research position with Jos Oomens at the FELIX facility in The Netherlands. In 2010 he took a postdoctoral appointment studying molecular collision dynamics with Dave Chandler at Sandia National Laboratories, where he joined the Combustion Research Facility as a staff scientist in 2013. His newest research interests lie in analytical methods development for monitoring chemically reactive systems.

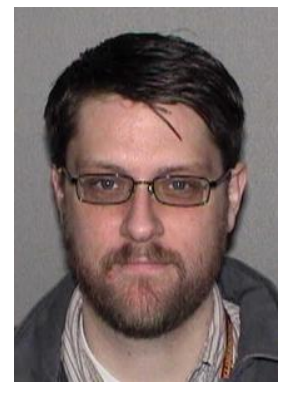

Ahren W. Jasper received a B.A. from Gustavus Adolphus College in 1998 and a Ph.D. from the University of Minnesota in 2003, where his advisor was Donald Truhlar. After a postdoctoral position at Argonne National Laboratories working with Stephen Klippenstein, he joined the staff of the Combustion Research Facility at Sandia National Laboratories in 2007 working as a theoretical combustion chemist. He studies gas phase dynamics, with an emphasis on nonadiabatic and pressure-dependent kinetics.

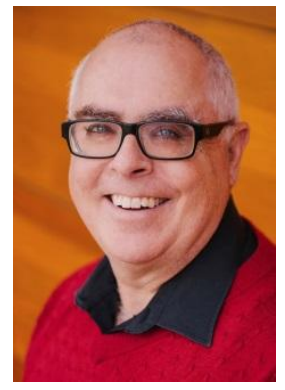

David W. Chandler received his B.S. in Chemistry at Univ. of New Mexico and a Ph.D. in Chemistry at Indiana University. After a postdoctoral fellowship with Dr. Richard N. Zare of Stanford University (1980-1982) he pursued his interest in photochemistry of small molecules as a staff scientist at Sandia National Laboratory in Livermore, California. In collaboration with Dr. Paul Houston (Cornell University), he developed the technique of Ion Imaging for which he was elected Fellow of American Physical Society, 1998, and received the Herbert P. Broida Award from the American Physical Society, 2001. He has been a Senior Scientist at Sandia since 2009. His use of the ion imaging technique to study both unimolecular dissociation and bimolecular scattering led his to discover a new technique for the cooling of molecules, Kinematic Cooling. Dave Chandler has published over 100 papers in the area of gas phase physical chemistry and is presently an Associate Editor of the Journal of Chemical Physics and Chairman of the Division of Chemical Physics of the American Physical Society. 


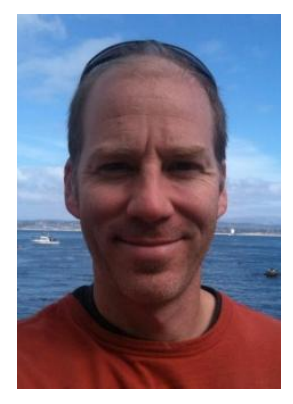

Jeffrey D. Steill received his B.S. in Chemistry and then Ph.D. in Chemical Physics from The University of Tennessee in 2007 with Prof. Robert N. Compton. He continued research in ion spectroscopy in a postdoctoral research position with Jos Oomens at the FELIX facility in The Netherlands. In 2010 he took a postdoctoral appointment studying molecular collision dynamics with Dave Chandler at Sandia National Laboratories, where he joined the Combustion Research Facility as a staff scientist in 2013. His newest research interests lie in analytical methods development for monitoring chemically reactive systems.

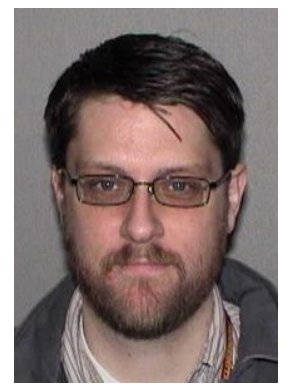

Ahren W. Jasper received a B.A. from Gustavus Adolphus College in 1998 and a Ph.D. from the University of Minnesota in 2003, where his advisor was Donald Truhlar. After a postdoctoral position at Argonne National Laboratories working with Stephen Klippenstein, he joined the staff of the Combustion Research Facility at Sandia National Laboratories in 2007 working as a theoretical combustion chemist. He studies gas phase dynamics, with an emphasis on nonadiabatic and pressure-dependent kinetics.

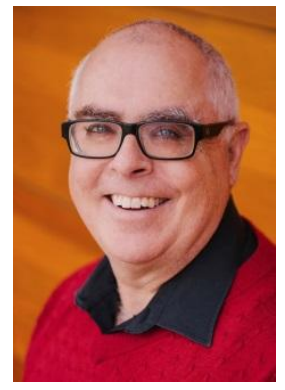

David W. Chandler received his B.S. in Chemistry at Univ. of New Mexico and a Ph.D. in Chemistry at Indiana University. After a postdoctoral fellowship with Dr. Richard N. Zare of Stanford University (1980-1982) he pursued his interest in photochemistry of small molecules as a staff scientist at Sandia National Laboratory in Livermore, California. In collaboration with Dr. Paul Houston (Cornell University), he developed the technique of Ion Imaging for which he was elected Fellow of American Physical Society, 1998, and received the Herbert P. Broida Award from the American Physical Society, 2001. He has been a Senior Scientist at Sandia since 2009. His use of the ion imaging technique to study both unimolecular dissociation and bimolecular scattering led his to discover a new technique for the cooling of molecules, Kinematic Cooling. Dave Chandler has published over 100 papers in the area of gas phase physical chemistry and is presently an Associate Editor of the Journal of Chemical Physics and Chairman of the Division of Chemical Physics of the American Physical Society. 

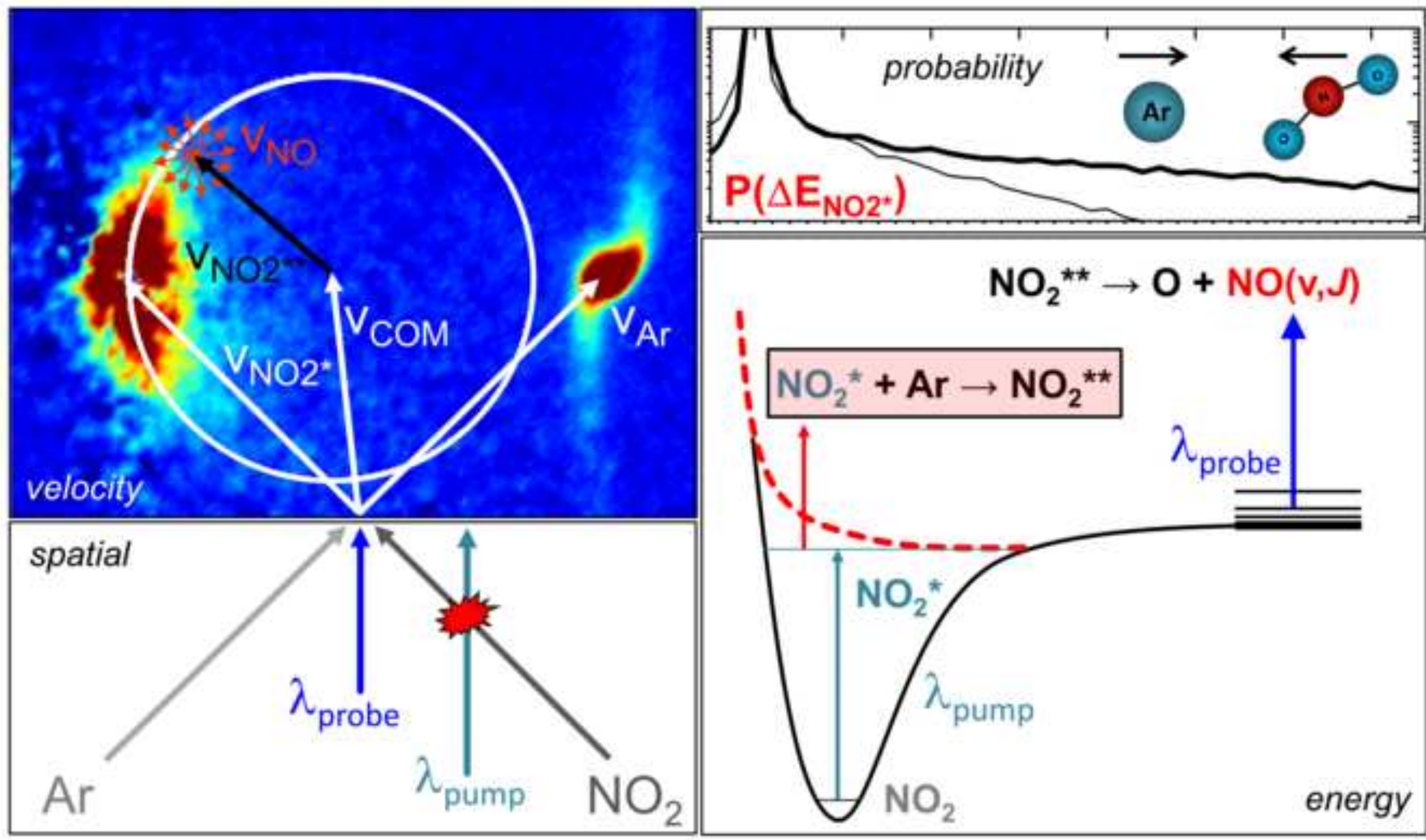